\title{
Die Zeit der großen Gräben: Modelle zur Chronologie des Michelsberger Fundplatzes von Heilbronn-Klingenberg „Schlossberg“, Stadtkreis Heilbronn, Baden-Württemberg
}

\author{
Ute Seidel,,$^{1}$ Elisabeth Stephan, ${ }^{2}$ Hans-Peter Stika, ${ }^{3}$ Elaine Dunbar, ${ }^{4}$ Bernd Kromer, ${ }^{5}$ Alex Bayliss, ${ }^{6}$ Nancy \\ Beavan, Frances Healy ${ }^{8}$ und Alasdair Whittle ${ }^{8}$
}

\author{
1 Landesamt für Denkmalpflege im Regierungspräsidium Stuttgart, Ref. 84.2, Dienstsitz Freiburg, Günterstalstrasse \\ 67, D-79100 Freiburg / Breisgau \\ 2 Landesamt für Denkmalpflege im Regierungspräsidium Stuttgart, Ref. 84.1, Dienstsitz Konstanz, \\ Stromeyersdorfstrasse 3, D-78467 Konstanz \\ 3 Institut für Botanik, Universität Hohenheim, Garbenstraße 30, D-70599 Stuttgart \\ 4 SUERC Radiocarbon Laboratory, Scottish Enterprise Technology Park, Rankine Avenue, East Kilbride G75 1QF, \\ $\mathrm{UK}$ \\ 5 Klaus-Tschira-Archäometrie-Zentrum, C4.8, D-68159 Mannheim \\ 6 Scientific Dating Team, Historic England, 1 Waterhouse Square, 138-42 Holborn, London EC1N 2ST, UK \\ 7 Department of Anatomy and Structural Biology, Otago School of Medical Sciences, University of Otago, PO Box \\ 913, Dunedin, Neuseeland \\ 8 Department of Archaeology and Conservation, Cardiff University, John Percival Building, Colum Drive, Cardiff \\ CF10 3EU, UK
}

\section{Erdwerke der Michelsberger Kultur}

Erdwerke sind ein herausragendes Phänomen der Michelsberger Kultur, die als archäologische Einheit im späten fünften und frühen vierten Jahrtausend cal BC in weiten Teilen Mitteleuropas verbreitet war. Für die Erbauer müssen die Erdwerke eine besondere Funktion und Bedeutung gehabt haben. Immerhin musste in den Bau einer solchen Anlage eine beträchtliche Arbeitsleistung investiert werden und oft scheinen Anlagen dieser Art, einmal erbaut, den Mittelpunkt intensiver Aktivitäten gebildet zu haben. In der Siedlungsorganisation der benachbarten Kulturgruppen dieser Zeit finden sich keine Erdwerke (Matuschik 1991). Für das Siedlungssystem der Michelsberger Kultur (MK) gibt es Hinweise darauf, dass immer nur kleine Gruppen an einem Platz siedelten und vielleicht nicht sehr lange an einem Ort blieben (Seidel 2008a, 388). Im Gegensatz dazu hielten die zeitgleichen Gemeinschaften, die die Tradition der mittelneolithischen Rössener Kultur fortführten, die sog. „Epirössener Gruppen“, lange genug an einem Platz fest, um Ansammlungen von über tausend Gruben zu hinterlassen. An einem Fundplatz der MK finden sich aber gewöhnlich nur etwa 5 bis 6 Gruben, die einer keramischen Stilstufe zuzuordnen sind (Seidel 2004 und 2008a, 388).

Für eine solch eher mobile und vielleicht segmentär organisierte Gesellschaft könnten Erdwerke als Versammlungsplätze gedient haben, und die Gründe für solche Zusammenkünfte können vielfältig gewesen sein. Häufig liegen die Erdwerke der MK in topographisch hervorgehobenen Lagen, an durch die Natur vorgegebenen Wegetrassen, oft auch an historisch belegten Straßen (Geschwinde 2013, 199). Für das Braunschweiger Land in Niedersachsen wurde ein Modell 
entwickelt, dem zufolge Erdwerke als Plätze für den Austausch von Rindern fungiert hätten (Geschwinde und Raetzel-Fabian 2009). Jedoch kann auf Grundlage der bekannten archäozoologischen Daten für die MK keine Bevorzugung der Rinderzucht attestiert werden (Stephan 2008, bes. 235ff.; Steppan 2003). Die Funktion der Erdwerke dürfte breiter gefasst gewesen sein und man kann durchaus unterschiedliche Zusammenkünfte und Anlässe sowohl mit wirtschaftlichem als auch rituellem Charakter in Betracht ziehen (Seidel 2008a). Unabhängig von der jeweils bevorzugten Interpretation ist aber der Versuch, eine präzisere Chronologie aufzubauen, eine der besten Möglichkeiten, neue Erkenntnisse zur Nutzung der Erdwerke zu gewinnen (vgl. Whittle et al. 2011). Der vorliegende Beitrag hat sich dies, am Beispiel von Klingenberg in Südwestdeutschland, zum Ziel gesetzt.

In Südwestdeutschland sind seit der Linearbandkeramik Erdwerke belegt, während des folgenden Mittelneolithikums fehlen sie dann aber fast ganz. Erst ab dem späten fünften Jahrtausend cal BC tritt die MK erneut mit charakteristischen Grabenwerken in Erscheinung. Das Erdwerk von Heilbronn-Klingenberg ist eine von etwa 100 bekannten Anlagen im Verbreitungsgebiet der MK (Jeunesse und Seidel 2010).

Die MK wird namentlich über das Vorkommen unverzierter, rundbodiger Keramik definiert, die durch Lüning in klassischer Weise in fünf typologische Stufen gegliedert wurde (Lüning 1967). Derzeit wird von einer Entstehung der MK im späten fünften Jahrtausend cal BC im östlichen Pariser Becken ausgegangen, von wo aus sich die MK nach Osten über den Großteil Deutschlands und bis nach Böhmen ausgebreitet habe. Ihr geschätztes Ende wird in der zweiten Hälfte des 37. Jahrhunderts cal BC angesetzt (Biel et al. 1998).

Erdwerke der MK können aus bis zu fünf Grabenringen bestehen, die in unregelmäßigen Abständen von Erdbrücken unterbrochen werden (Seidel 2008a; Jeunesse und Seidel 2010; vgl. Andersen 2015; Petrasch 2015). Vielfach dokumentierte Spuren von Einbauten, wie Pfostenlöcher und Schlitzgruben in diesen Übergängen, lassen an ausgebaute Tore denken. In einigen Fällen wurden Spuren einer Palisade innerhalb der Gräben beobachtet. Zumindest für Klingenberg ist gesichert, dass hinter dem innersten Graben auch ein Wall aufgeschüttet war, doch ist seine genaue Position unklar. In verschiedenen Erdwerken fanden sich entlang der Grabenstrecken Reste grabenbegleitender Konstruktionen, wie verbrannte Hölzer, oder Steine von Trockenmauerwerk. Das Fundmaterial aus den Gräben variiert von Fundplatz zu Fundplatz, vielfach sind aber Skelettelemente von Tieren und Menschen darunter (Andersen 2015; Seidel 2008a). Wo größere Areale ausgegraben wurden, wurden zusätzlich zu den Erdwerksgräben bei guter Erhaltung auch Gruben freigelegt. Die Gruben ergaben ein breites Spektrum an Funden, neben Resten aus dem Alltagsleben, wie Keramik und Tierknochen, auch Gegenstände aus dem nicht-alltäglichen Bereich, wie ein Jadeitbeil, Miniaturbeile aus Ton, verziegelter Wandverputz in Form weiblicher Brüste in Klingenberg, oder ehemals aufgespießte Schädel oder eine Gesichtsmaske. Zu den nicht alltäglichen Funden sind auch Deponierungen von Tieren und Menschen zu zählen.

Nur zwei der Erdwerke der MK - Urmitz und Wiesbaden-Schierstein am Mittelrhein erreichten beeindruckende Größen von respektive 60 ha und fast 100 ha. Die meisten Erdwerke der MK umfassen hingegen Innenräume von 10 bis 40 ha; einige wenige Anlagen bleiben unter 10 ha, von diesen ist Klingenberg mit seinen ca. 2,5 ha Innenfläche wohl das kleinste (Seidel 
2008a, 357, Tab. 42). Im Verlauf der MK zeichnet sich ein Trend ab, der von frühen, größeren Grabenwerken mit häufigeren Unterbrechungen, die in Flusstälern oder auf niedrigen Hügeln liegen, zu den kleineren Erdwerken der späteren MK führt, welche an topographisch markanten und potentiell defensiven Standorten angelegt wurden (Matuschik 1991; Seidel 2008a; Geschwinde 2013). Das namengebende Erdwerk auf dem „Michelsberg“, umfasst allerdings mehr als 10 ha und dominiert die höchste Erhebung des Kraichgaus über dem Rheintal; es wurde wohl erst zur Stufe MK IV angelegt, so dass diese Entwicklung nicht ganz linear verlaufen sein dürfte.

In zwei Aspekten profitiert die Untersuchung der MK Erdwerke ohne Zweifel von einer präziseren Datierung: in der Festlegung des zeitlichen Ablaufs und in der Untersuchung der Dauer ihrer Nutzungsphase(n). Dies sollte idealerweise auf regionaler, oder auf noch großräumigerer, Ebene für mehrere Anlagen untersucht werden (Whittle et al. 2011). Im vorliegenden Beitrag beginnen wir dieses Vorhaben zunächst mit dem Fallbeispiel Klingenberg.

\section{Das Erdwerk von Klingenberg}

Die kleine Abschnittsbefestigung von Heilbronn-Klingenberg „Schlossberg“, Stadtkreis Heilbronn in Baden-Württemberg (Abb. 1), datiert in die späteste Stufe der Michelsberger Kultur (MK V oder Fazies Munzingen B). Die Laufzeit dieser Stufe wird auf 3750-3650 cal BC angesetzt (Seidel 2008a, 315-26; siehe Abschnitt „Diskussion“). Das Erdwerk wurde 1985 im Luftbild entdeckt und 1986/7 von Jörg Biel komplett untersucht. Es ist damit bis heute das einzige Erdwerk der MK, bei dem nicht nur das Grabensystem und die Innenfläche, sondern auch die Fläche außerhalb der Gräben untersucht wurde. Zwei parallel geführte Gräben riegeln den „Schlossberg“, einen Lössvorsprung zwischen dem Neckar und einem kleinen Zufluss, dem Klingenbach, oberhalb einer historischen Neckarfurt ab. Die Gräben umschließen eine Innenfläche von etwa 2,5 ha, mit der Fläche außerhalb der Gräben sind insgesamt ca. 3 ha mit Gruben bedeckt.

Bei den Grabungen wurden rund 650 Gruben, zwei Gräben, einige Schlitzgruben und Pfostenlöcher in der Nähe von Erdbrücken, sowie der $7 \mathrm{~m}$ lange Abschnitt einer „Palisade“ innerhalb des inneren Grabens dokumentiert (Abb. 2; Seidel 2008a). Zu den bemerkenswertesten Befunden gehören die sehr gut erhaltenen Überreste hölzerner Konstruktionen, Pfosten und Bretter, die brennend in einige Segmente der beiden Gräben gestürzt waren und den umgebenden Löss orange verziegelten (Abb. 3). In den Gräben von Klingenberg wurden keine menschlichen Überreste entdeckt, dafür konnten in den Gruben und Gräben die Skelette bzw. Teilskelette (TS) von 35 Hunden und 12 anderen Haus- und Wildtierarten dokumentiert werden (Abb. 4; Stephan 2008).

Die von Seidel (2008a, 246-99, 315-26) erarbeitete Chronologie des Fundplatzes basiert auf einer Clusteranalyse der Profile aller rekonstruierbaren Gefäße und einer Seriation der resultierenden Gefäßtypen, den Keramiktypologien nach Lüning (1967) und Höhn (2002) und den zum damaligen Zeitpunkt verfügbaren 25 Radiokarbondaten. Die auf dieser Grundlage vorgeschlagene Michelsberger Belegung des Fundplatzes begann - einigen Scherben zufolge während MK II, d.h. in den Jahrzehnten vor 4000 cal BC. Eine weitere Aktivitätsphase, der sich 
Material aus sechs Gruben zuordnen ließ, datiert nach MK III/IV, d.h. die Jahrzehnte nach 4000 cal BC. Es wurde davon ausgegangen, dass nach dieser zweiten Belegung zu Beginn von MK V/Munzingen, also im 38. Jahrhundert cal BC das gesamte Areal - allerdings noch ohne Grabenwerk - von hunderten von Gruben bedeckt wurde. Der Großteil der Keramik von Klingenberg entspricht typologisch MK V/Munzingen. In dieser Zeit fand ein Wandel der Keramik statt, insofern flachbodige Formen, wie sie Munzingen B kennzeichnen, in das Keramikspektrum der MK aufgenommen wurden (Seidel 2008a-b). Um 3700 cal BC wurden nach diesem Modell die beiden Erdwerksgräben ausgehoben, die dann das vormals genutzte Areal durchtrennten. Es wurde angenommen, dass nach dem Bau der Gräben nur noch der Innenbereich besiedelt war. Die Gräben wurden als zeitgleich angesehen, da sie eine vergleichbare Form und Verfüllstruktur aufwiesen, die gleiche Keramik enthielten und übereinstimmende ${ }^{14} \mathrm{C}$-Daten ermittelt wurden. Als Zeitpunkt der Zerstörung der Grabenanlage, d.h. des Ereignisses, das zum Brand der zugehörigen Holzkonstruktionen führte, wurden die Jahre nach 3700 cal BC angenommen.

Allerdings blieben viele dieser Schlussfolgerungen vorläufig und die Fundstelle warf nach wie vor Fragen auf, die sowohl fundplatzspezifisch sind als auch den weiteren Kontext der MK betreffen:

- Über welche Zeitspanne erstreckte sich die Besiedlung von Klingenberg insgesamt-?

- Wurde der Platz kontinuierlich oder nur episodisch genutzt? In letzterem Fall wäre zu fragen, wie viele unterschiedliche Besiedlungsphasen es gab.

- Wie war das zeitliche Verhältnis zwischen Erdwerk und Gruben? Wurden die Bereiche innerhalb und außerhalb des Grabenwerkes jeweils vor und/oder nach seiner Erbauung genutzt?

- Gehören alle Hundedeponierungen in den gleichen Aktivitätshorizont?

- Bedeutete die Zerstörung des Grabenwerks zugleich auch das Ende der Nutzung bzw. Besiedlung des Platzes?

- Welchen Beitrag kann eine präzisere/genauere Chronologie für Klingenberg für unser Verständnis der Erdwerke der MK im Besonderen und für die Entwicklung der Keramiktypologie der MK im Allgemeinen leisten?

Diese Fragen waren der Anstoß, das hier vorgelegte Datierungsprogramm im Rahmen des Projektes The Times of Their Lives (ToTL; siehe Danksagung) durchzuführen. Das Projekt ToTL verfolgt das Ziel, die Chronologie ausgewählter Fundplätze und zugleich Aspekte des europäischen Neolithikums präziser zu fassen, indem nach rigorosen Kriterien ausgewähltes Material ${ }^{14} \mathrm{C}$-datiert und unter Anwendung der bayes'schen Statistik analysiert wird. Präzisere Datierungen ermöglichen es, den Besiedlungsrhythmus verschiedener Fundplätze, die Nutzungszeit von Monumenten, wie auch Traditionen oder Phänomene von Kontinuität und Diskontinuität besser zu beleuchten (Whittle et al. 2008).

\section{Naturwissenschaftliche Datierung}


Aktuell liegen 94 Radiokarbonmessungen an Proben von Klingenberg vor (Tabellen 1 und 2). Unter ihnen sind acht Paare von Kontrollmessungen (also unabhängige ${ }^{14} \mathrm{C}$-Messungen an zwei Teilen der gleichen Probe) und zwei Gruppen von jeweils drei Kontrollmessungen. Dreißig Messungen wurden im Zuge der ursprünglichen Analyse und Publikation des Fundplatzes durchgeführt, zehn im Radiokarbon-Labor der Heidelberger Akademie der Wissenschaften (Hd), weitere 20 im Leibniz-Labor der Christian Albrechts Universität in Kiel (KIA-). Nach der Publikation 2008 wurde eine weitere Probe im Curt-Engelhorn-Zentrum Archäometrie in Mannheim (MAMS-) analysiert. Im Zuge des ToTL-Projektes wurden weitere 63 Messungen durchgeführt, davon 31 am Curt-Engelhorn-Zentrum Archäometrie und 32 am Scottish Universities Environmental Research Centre, East Kilbride (SUERC-). Von diesen 94 Datierungen beziehen sich 78 auf die Michelsberger Nutzung des Fundplatzes. Zwei verkohlte Getreidekörner aus dem äußeren Graben erbrachten kein Ergebnis.

Probenaufbereitung und Messung

Die in Heidelberg gemessenen Knochenproben wurden mit Salzsäure demineralisiert und dann mehrere Tage in Dialyseschläuchen aufbewahrt, um vor der Verbrennung Proteine kurzer Kettenlänge $(<10 \mathrm{kD})$ zu entfernen. Das resultierende $\mathrm{CO}_{2}$ wurde gereinigt und sieben Tage in Hochdruck-Gas-Proportionalzählrohren gemessen (Münnich 1957; Schoch et al. 1980; Dörr et al. 1989, Kromer und Münnich 1992).

Alle übrigen Daten wurden mittels Beschleuniger-Massenspektrometrie (AMS) datiert. In Kiel wurden verkohlte Pflanzenreste und Holzkohle einer Säure-Lauge-Säure Vorbehandlung unterworfen (Mook und Waterbolk 1985). Pulverisierte Knochenproben wurden erst mit Azeton behandelt, dann mit deionisiertem Wasser gespült und schließlich in Salzsäure (1 \%) demineralisiert (Grootes et al. 2004). Um die mobileren Huminsäuren zu entfernen, wurde der demineralisierte Knochen mit Natriumhydroxid ( $1 \%$ bei $20^{\circ} \mathrm{C}$ für eine Stunde) und dann nochmals mit Salzsäure (1\% bei $20^{\circ} \mathrm{C}$ für eine Stunde) behandelt. Die so gewonnene Knochengelatine wurde über Nacht in Wasser aufgelöst (bei $85^{\circ} \mathrm{C}$ und $\mathrm{pH}$ 3), durch einen ausgeglühten Silberfilter mit $0,45 \mu \mathrm{m}$ Porenweite gefiltert und gefriergetrocknet. Glühen, Graphitisierung und Messung verliefen wie von Nadeau et al. $(1997 ; 1998)$ beschrieben.

In Mannheim wurde verkohltes Getreide einer Säure-Lauge-Säure Vorbehandlung unterworfen (Mook und Waterbolk 1985); das aus den Knochenproben gewonnene Kollagen wurde ultrafiltriert und gefriergetrocknet (Brown et al. 1988) bevor es zu $\mathrm{CO}_{2}$ verbrannt und graphitisiert und schließlich, wie von Kromer et al. (2013) beschrieben, AMS-datiert wurde.

In East Kilbride wurden Proben an verkohlten Pflanzen wie von Mook und Waterbolk (1985) beschrieben vorbehandelt und Knochenproben mittels Gelatinisierung und Ultrafiltration behandelt (Brock et al. 2010). Alle Proben wurden zu Kohlendioxid verbrannt (Vandeputte et al. 1996), graphitisiert (Slota et al. 1987) und AMS-datiert (Freeman et al. 2010).

In Heidelberg und East Kilbride wurden $\delta^{13} \mathrm{C}_{-}-$Werte mittels IsotopenverhältnisMassenspektrometrie (IRMS) an Teilproben des zur Datierung verbrannten Kohlendioxids gewonnen. Diese Werte wurden zur Bestimmung des konventionellen ${ }^{14} \mathrm{C}$-Alters (Stuiver und 
Polach 1977) genutzt und spiegeln vor allem die natürliche Isotopenzusammensetzung des datierten Materials wieder. Sie lassen sich daher auch zur Analyse der Ernährung nutzen. In Mannheim und Kiel wurden die $\delta{ }^{13} \mathrm{C}$-Werte durch Beschleuniger-Massenspektrometrie (AMS) an den zur Datierung genutzten Graphit-Targets gemessen. Diese Werte wurden bei der Berechnung des konventionellen ${ }^{14} \mathrm{C}$-Alters (Stuiver und Polach 1977) genutzt, können aber, zusätzlich zur natürlichen Isotopenzusammensetzung, eine während der Aufbereitung und Messung eingeführte Fraktionierungskomponente enthalten und daher nicht zu einer Analyse der Ernährung herangezogen werden. Daher wurden für die im Zuge des ToTL-Projekts eingereichten Knochenproben die stabilen Isotope Kohlenstoff und Stickstoff mittels Isotopenverhältnis-Massenspektrometrie (IRMS) an Teilproben des zur Datierung aufbereiteten Kollagens gemessen. Im SUERC Labor wurden die $\delta^{13} \mathrm{C}$ - und $\delta^{15} \mathrm{~N}$-Proben wie von Sayle et al. (2014) beschrieben aufbereitet und analysiert. Teilproben der im MAMS-Labor aufbereiteten und datierten Gelatine wurden an der Isotrace facility, University of Otago Chemistry Department auf $\delta^{13} \mathrm{C}$ und $\delta^{15} \mathrm{~N}$ untersucht. Dabei wurden die von Beavan Athfield et al. $(2008,3)$ beschriebenen Methoden angewendet. Diese Messungen fließen in die unten beschriebene Analyse der Ernährung auf Grundlage der stabilen Isotope ein.

Qualitätskontrolle

Alle vier Labore nehmen an laufenden Programmen zur internen Qualitätskontrolle und an internationalen Ringvergleichen teil (Scott 2003; Scott et al. 2007; 2010a-b).

Die Konsistenz der Ergebnisse wurde im Laufe des ToTL-Projektes zusätzlich mittels wiederholter Messung der gleichen Probe durch unterschiedliche Labore überprüft. Von zehn Sätzen Kontrollmessungen, die an einzelnen Knochenstücken in Mannheim und East Kilbride durchgeführt wurden, sind sechs bei $95 \%$ Wahrscheinlichkeit statistisch konsistent (Ward und Wilson 1978), die verbleibenden vier Messungen sind es nicht (Tabelle 2). Als wir die inkonsistenten Ergebnisse erhielten (Frühjahr 2014), wurden beide Labore angefragt, die betreffenden Proben noch einmal zu datieren. Jedoch war nur für zwei der Proben noch Material vorhanden, das in Mannheim noch einmal datiert werden konnte. Die Ergebnisse waren mit den ersten MAMS Ergebnissen statistisch konsistent, und damit nach wie vor inkonsistent mit den SUERC Ergebnissen (Tabelle 2: SUERC-52388, MAMS-20318.1-2; SUERC-52420, MAMS20332.1-2). Keines der Labore konnte einen Fehler bei der Datenverarbeitung entdecken. Von den acht Gruppen aus Kontrollmessungen, die im Frühjahr und Sommer 2014 gemessen wurden, sind vier statistisch konsistent, in den anderen vier Fällen sind die Daten aus Mannheim etwas jünger als die aus East Kilbride. Die beiden im Herbst 2014 gemessenen Gruppen sind mit $95 \%$ Wahrscheinlichkeit statistisch konsistent. Wir können nur davon ausgehen, dass Anfang 2014 in einem oder beiden Laboren ein Fehler aufgetreten ist, der zu einem leichten Offset in einigen ihrer Ergebnisse geführt hat. Es ist unmöglich zu sagen, wo die Ungenauigkeit begründet liegt. Beide Datenserien sind konsistent mit den stratigraphischen Verhältnissen und den anderen in das Modell einbezogenen Informationen.

Alle ermittelten Ergebnisse fließen in die unten vorgestellten Modelle mit ein, da wir nicht der Ansicht sind, dass die leichten Offsets in einigen der Ergebnisse einen großen Einfluss auf die 
von den Modellen errechneten A-posteriori-Wahrscheinlichkeitsverteilungen nehmen und es keine wissenschaftlich oder archäologisch begründeten Argumente dafür gibt, den einen oder anderen Datensatz zu bevorzugen. Wo auch immer der Grund für die beobachteten Offsets liegt, er hat offenbar nur die Hälfte der Anfang 2014 an einem der Labore gemessenen Knochenproben beeinflusst und betrifft damit nur acht oder neun der 78 Ergebnisse aus Michelsberger Befundzusammenhängen. Den Auswirkungen der leichten Offsets in diesen Proben kann durch gewichtete Mittelwerte (Ward und Wilson 1978) der Kontrollmessungen - die für etwa die Hälfte der angenommen Fälle vorliegen - entgegengewirkt werden, sowie durch die überwältigende Mehrzahl der Daten im Modell, die nicht davon beeinflusst wird. Auch die durch das Modell auferlegten Bedingungen schwächen die etwaigen Auswirkungen ab, die die beobachteten Offsets auf einen kleinen Anteil der Ergebnisse haben könnten.

Kontrollmessungen in diesen beiden Laboren, die später im Laufe des ToTL-Projekts an Proben von anderen Fundplätzen durchgeführt wurden, lagen immer im Rahmen der statistischen Erwartungen.

\section{Chronologische Modelle}

Das neue Datierungsprogramm für Klingenberg wurde von Anfang an im Hinblick auf eine Bayes'sche Analyse entworfen (Buck et al. 1996; Bayliss 2009). Das Prinzip der bayes'schen Herangehensweise an die Interpretation von Daten beruht auf dem Satz von Bayes (Bayes 1763). Dieser besagt, dass neue Daten, die zu einem Problem gesammelt werden (die „standardisierten Likelihoods"), im Kontext bestehender Erfahrungen und Vorwissen zu diesem Problem (, $A$ priori-Wahrscheinlichkeit“, oder Prior) analysiert werden. Die Kombination beider kann zu einem neuen Verständnis des Problems („A-posteriori-Wahrscheinlichkeit“, oder Posterior) führen, das dann wieder zum Vorwissen für ein späteres Modell werden kann. Die bayes'sche Analyse kombiniert also archäologische Informationen und Informationen aus $\operatorname{der}{ }^{14} \mathrm{C}$-Datierung, indem beide als Wahrscheinlichkeitsdichtefunktionen ausgedrückt werden. In dieser Form werden auch die Posteriore beschrieben.

Wenn Modelle archäologischer Chronologien entworfen werden, bilden kalibrierte

Radiokarbondatierungen die Komponente der „standardisierten Likelihoods“ des Modells, während die Archäologie die „Priore“ beisteuert. Auf diese Weise werden die Radiokarbondaten im Licht archäologischer Informationen neu interpretiert, um Posteriore zu diesen Daten zu generieren. Solche Schätzungen ändern sich mit dem/den angewendeten Modell(en), und verschiedene Modelle können auf der Grundlage unterschiedlicher Interpretationen der gleichen Daten entworfen werden (Bayliss et al. 2007). Das Ziel der Modellbildung ist es, von den Zeitpunkten, an denen die einzelnen Proben den Kohlenstoffzyklus verlassen haben, zu einer Datierung der mit diesen Proben assoziierten archäologisch fassbaren Ereignisse zu gelangen.

Priore fallen in zwei große Gruppen: Informative und nicht informative. Informative Priore, die bei der Modellbildung mit Daten aus archäologischen Zusammenhängen verwendet werden, basieren oft auf den stratigraphischen Zusammenhängen zwischen den Schichten, aus denen die einzelnen Proben stammen. In Abbildung 11 werden beispielsweise die Daten aus Kontext 435 durch die existierende Information eingeschränkt, dass SUERC-52406 früher ist als SUERC- 
52401 und MAMS-20320. Ein oft verwendeter nicht informativer Prior ist, dass die datierten Proben eine mehr oder weniger kontinuierliche Aktivitätsphase abdecken, wie beispielsweise den Bau und die Nutzung des MK V/Munzingen Erdwerkes und der zugehörigen Gruben (Abb. 7), und mehr oder weniger gleichmäßig über diese Phase verteilt sind, ohne dass sie deswegen zwangsläufig das älteste oder jüngste Material dieser Phase mit einschließen müssen (Buck et al. 1992). Diese Annahme ist notwendig, um die den Radiokarbondatierungen innewohnende Streuung einzuschränken, die andernfalls dazu führen würde, dass Aktivitätsepisoden so wirken, als würden sie früher beginnen, länger anhalten und später enden, als es eigentlich der Fall war (Steier und Rom 2000). In diesem Sinn bezeichnet „Phase“ lediglich eine Gruppe zusammenhängender Daten ohne innere Abfolge.

Das Modell wird in OxCal definiert, wobei die einzelnen ${ }^{14} \mathrm{C}-\mathrm{D}$ aten und die bekannte relativFormatted: Superscript chronologische Abfolge der Proben eingeht. Nachdem die kalibrierten Wahrscheinlichkeitsverteilungen der einzelnen ${ }^{14} \mathrm{C}$-Alter errechnet worden sind, versucht das Programm, diese Verteilungen mit der A-priori-Information in Einklang zu bringen, indem für jede Verteilung wiederholt Stichproben gezogen werden, um eine mit der Modellstruktur konsistente Reihe an Lösungen aufzubauen. Dies geschieht durch ein zufälliges Stichprobenverfahren (Markov Chain Monte Carlo oder MCMC), das einen repräsentativen Satz möglicher Datierungen generiert. Dieser Prozess produziert für das kalendarische Alter jeder Probe eine a-posteriori-_Dichteverteilung, die nur einen Teil der kalibrierten Wahrscheinlichkeitsverteilung einnimmt. In den Abbildungen in diesem Bericht sind die $a$ posteriori_-Dichteverteilungen in einem dunkleren und die kalibrierten Radiokarbondaten, aus denen die Stichproben genommen wurden, in einem helleren Farbton dargestellt. Das Modell kann auch a-posteriori_-Verteilungen für Ereignisse errechnen, die nicht direkt mit bestimmten Radiokarbondatierungen verknüpft sind. Dazu gehören Schätzungen für den Anfang und das Ende von Aktivitätsphasen (z.B. Abb. 13: Beginn $M K V / M_{z}$ Gruben außerbalb des Erdwerks). Durch Errechnen der Differenz zwischen solchen Parametern können die Dauer bestimmter Aktivitätsphasen (also die Differenz zwischen deren Anfang und Ende (z.B. Abb. 17: Nutzung $M K V / M z$ Gruben außerhalb des Erdwerks) und die Intervalle zwischen bestimmten Ereignissen (z.B. Abb. 18: Gruben Innenraum / Gruben Außenraum) abgeschätzt werden. In letzterem Fall können diese Schätzungen teilweise negativ ausfallen, wenn die Abfolge der datierten Ereignisse selbst unklar ist.

Die höchsten a-posteriori_-Dichteintervalle, also die Datierungsspannen, die die a-posterioriVerteilungen zu einem bestimmten Wahrscheinlichkeitsgrad beschreiben, werden konventionellerweise kursiv gedruckt (z.B. „3790-3675 cal BC") um sie klar von einfachen kalibrierten Radiokarbondaten zu unterscheiden. Jede a-posteriori-Verteilung hat einen Parameternamen, der auch kursiv gedruckt ist. In manchen Fällen (z.B. Beginn $M K V / M z$ Gruben außerbalb des Erdwerks) sind dies spezifische Bezeichnungen, aber sonst dient der Kursivdruck dazu, die a-posteriori-Verteilung einer bestimmten ${ }^{14} \mathrm{C}$-Probe (z.B. MAMS-20317) und die einfache kalibrierte ${ }^{14} \mathrm{C}$-Datierung dieser Probe (also MAMS-20317) zu unterscheiden.

Die von OxCal errechneten statistischen Werte geben einen Hinweis auf die Verlässlichkeit eines Modells. Einer ist der individuelle Übereinstimmungsindex, der die Kompatibilität der a-priori_ und a-posteriori-Verteilungen ausdrückt (z.B. Abb. 13: MAMS-20137 [A: 100]). Liegt die $a$ posteriori_-Verteilung in einer Region der a-priori_-Verteilung mit hoher Wahrscheinlichkeit, ist der 
Übereinstimmungsindex hoch (manchmal 100 oder höher). Fällt der Übereinstimmungsindex unter 60 (ein Schwellenwert, der der 95\% Signifikanz in einem $\chi^{2}$-Test entspricht), dann muss die Stellung dieser ${ }^{14} \mathrm{C}$-Datierung im Modell noch einmal überdacht werden. Einige Fälle niedriger individueller Übereinstimmung sind wegen der inhärenten statistischen Streuung der Radiokarbondaten zu erwarten. Aber ein sehr niedriger Übereinstimmungsindex könnte bedeuten, dass ein bestimmtes ${ }^{14} \mathrm{C}$-Ergebnis ein statistischer Ausreißer ist (mehr als zwei Standardabweichungen vom echten Radiokarbonalter der Probe entfernt), dass die Probe verlagert oder intrusiv ist (also dass das kalendarische Datum von dem abweicht, das von seiner stratigraphischen Position suggeriert wurde), oder dass die Probe mit externem Kohlenstoff verunreinigt wurde. Ein weiterer Übereinstimmungsindex, Amodel, wird aus den individuellen Übereinstimmungsindizes errechnet und zeigt an, ob das Modell insgesamt, auf Grundlage dieser Daten, wahrscheinlich ist. In den meisten Fällen hat dieser Index auch einen Schwellenwert von 60. In Abbildung 7 zeigt „Amodel: 89“ beispielsweise, dass die Radiokarbondaten gut zueinander und ins Modell passen. Jedoch ist Amodel: 110 nicht besser als Amodel: 61. Ein Modell mit Amodel: 61 ist lediglich stärker eingeschränkt.

Es kommt oft vor, dass die Anzahl der Daten, die in ein Modell mit einbezogen werden, größer ist als die Anzahl der Verteilungen, die in die Berechnungen einfließen (die effektiven Wahrscheinlichkeiten). Im Fall von Klingenberg hat das mehrere Gründe. Gibt es zwei oder mehr Datierungen der gleichen Probe, geht deren gewichtetes Mittel ins Modell ein, was durch den Befehl R_Combine ausgedrückt wird (z.B. Abb. 10: TS 16). Sind Datierungen zu ungenau, können sie vollständig von den Berechnungen ausgeschlossen werden, was durch ein ,?“ auf den Diagrammen ausgedrückt wird (z.B. Abb. 10: TS 38?).

Lindley (1985) bietet eine leicht verständliche Einführung in die Prinzipien der Bayes'schen Statistik und weitere archäologische Fallbeispiele werden von Buck et al. (1996) und Bayliss et al. (2007) vorgestellt. Eine vollständige Beschreibung der hier verwendeten Methoden findet sich bei Bronk Ramsey (1995; 2001; 2009), Bronk Ramsey und Lee (2013) und Bayliss et al. (2011).

\section{Auswahl der Proben}

Die Proben in diesem Projekt wurden nach den gleichen Kriterien ausgewählt, nach denen auch die existierenden Daten bewertet wurden. Diese Kriterien wurden bereits andernorts im Detail dargelegt (z.B. Bayliss et al. 2011, 38-42). Sie sollen sicherstellen, dass eine Probe gleichzeitig mit ihrem Auffindungskontext ist, und nicht etwa zum Zeitpunkt ihrer Einlagerung bereits alt war. Mit anderen Worten, zum Zeitpunkt an dem sie in den Boden gelangte, sollte eine Probe den Radiokarbonzyklus erst vor kurzer Zeit verlassen haben, und sie sollte in ihrem ursprünglichen Einbettungskontext aufgefunden und nicht verlagert worden sein.

Die Probenarten, die hauptsächlich für die Datierung ausgewählt werden, lassen sich — grob in absteigender Reihenfolge ihrer Verlässlichkeit — wie folgt zusammenfassen,:

- Knochen, die im anatomischen Zusammenhang aufgefunden wurden. Diese Proben wurden zum Zeitpunkt ihrer Einbettung noch von Weichteilen zusammengehalten und stammen daher von vor kurzem verstorbenen Individuen. 
- Knochen, die während der Analyse als artikulierende Knochen eines Individuums identifiziert wurden, vor allem dann, wenn ein Individuum durch zahlreiche Skelettteile repräsentiert ist. Diese Knochen könnten noch zusammenhängend in den Boden gelangt sein oder wurden vor ihrer Einbettung nur minimal gestört (Teilskelette).

- Knochen mit anpassenden, noch nicht verwachsenen Epiphysen, die während der Analyse identifiziert wurden (aus den oben dargelegten Gründen).

- Knochen- oder Geweihgeräte, die auf oder nahe der Sohle der Befunde zurückblieben, zu deren Aushebung sie verwendet worden waren. Zum Graben sind elastische, frische Geweihstücke nötig, das Geweih sollte also im gleichen Jahr (oder nur knapp vorher) abgeworfen worden sein, in dem es genutzt wurde. Vorausgesetzt, dass das Geweihgerät genau dort aufgefunden wurde, wo man es weggeworfen hatte, sollte sein Alter also dem des Befundes sehr ähnlich sein.

- Verkohle Speisereste, die an der Innenseite einer Gruppe von Scherben einer einzigen Gefäßeinheit anhaften. Hierbei handelt es sich höchstwahrscheinlich um Speisereste (und nicht um Verrußung durch das Brennmaterial) und es ist relativ wahrscheinlich, dass ein zu größeren Teilen vorhandenes Gefäß an dem Ort liegt, an dem es entsorgt wurde. Die Tatsache, dass die relativ empfindlichen Speisereste sich erhalten haben, weist ebenfalls darauf hin, dass die Scherben nicht übermäßig unter nachträglichen Störungen gelitten haben.

- Einzelne Fragmente verkohlter, kurzlebiger Pflanzenarten, die ursächlich mit ihrem Auffindungskontext zusammenhängen (z.B. Holzkohle aus einer Herdstelle oder einem Scheiterhaufen) oder die Teile von baulichen Strukturen sind (z.B. die Waldkante verkohlter Pfosten). Die Nutzung einzelner Fragmente verhindert das Risiko, Materialien unterschiedlichen Alters in der-selben Probe zu vermischen. Die Datierung von mehr als einer Probe pro Kontext ermöglicht es, vereinzelte Fragmente älteren Materials zu erkennen (Ashmore 1999).

- Einzelne Fragmente verkohlter, kurzlebiger Pflanzen aus zusammenhängenden Ansammlungen verkohlten Materials und bei denen auf Grund ihrer Geschlossenheit und Zerbrechlichkeit davon auszugehen ist, dass es sich um eine Primärdeponierung handelt (z.B. verkohltes Getreide aus einer größeren Konzentration in einer Grube).

Im Falle von Klingenberg besteht der Großteil der im Rahmen des ToTL-Projektes datierten Proben entweder aus verkohlten Getreideresten oder aus Tierknochen, die im anatomischen Verband aufgefunden oder als zusammengehörig definiert werden konnten (Tabelle 1). Die meisten dieser Knochenproben stammen von vollständig deponierten Tieren oder Teilskeletten (TS), meist von Hunden, aber auch von Rothirschen, Auerochsen und Hausrindern (Stephan 2008a, 185-93). Einige wenige nicht artikulierte Knochen, d.h. Einzelknochen, wurden dann datiert, wenn es den Anschein hatte, dass sie termini post quos für ihre Auffindungskontexte oder für den verschwundenen Wall des Erdwerkes liefern könnten. Keine der drei menschlichen Bestattungen Michelsberger Datums (Wahl 2008, 718-25) konnte aufgefunden werden, um als Ergänzung für das bereits zwischen der Publikation der Ausgrabung und dem Beginn des ToTLProjektes datierte Individuum (Abb. 13: MAMS-19231; Wahl 2008, 721-2, 725) zu fungieren. 
Von der ursprünglich zahlreich vorhandenen Holzkohle konnte kein einziges Stück mehr aufgefunden werden.

In allen hier beschriebenen Modellen werden Datierungen an Einzelnochen von Tieren, die zum Zeitpunkt ihrer Einbettung bereits alt gewesen sein könnten, sowie Datierungen an Proben, die potentiell älter als ihr Auffindungskontext sein könnten, als termini post quos für ihre

Auffindungskontexte verwendet, sofern sie sich nicht als konsistent mit anderen Daten an kurzlebigem Material aus dem gleichen Kontext erwiesen. Dazu wird die Funktion ,after“ genutzt (z.B. Abb. 13: KLA-22468).

\section{Ergebnisse und Kalibration}

The Radiokarbondatierungen und die zugehörigen Messungen sind in Tabelle 2 aufgelistet. Bei allen handelt es sich um konventionelle, um Fraktionierungseffekte korrigierte ${ }^{14} \mathrm{C}$-Alter (Stuiver und Polach 1977). In Tabelle 2 wurden die ${ }^{14} \mathrm{C}$-Ergebnisse mit einem Kurven-Schnittverfahren kalibriert (Stuiver und Reimer 1986); in den Abbildungen und für die bayes'schen Modelle wurde mit der Wahrscheinlichkeitsmethode kalibriert (Stuiver und Reimer 1993). Alle Kalibrierungen und die unten beschriebenen bayes'schen Chronologiemodelle wurden mit OxCal v4.2 (http://c14.arch.ox.ac.uk/) und der INTCAL 13 Kalibrationskurve (Reimer et al. 2013) erstellt. Die höchsten a-posteriori Intervalle wurden auf fünf Jahre aufgerundet. Viele der Daten fallen auf sukzessive Plateaus in der Kalibrationskurve, die im 39. und späten 38. Jahrhundert cal BC auftreten (Abb. 5), so dass ihre Verteilungen, vor allem im 39. Jahrhundert, auch nachdem sie von den Modellen eingeschränkt wurden, entsprechend breit und manchmal bimodal ausfallen. Die resultierende Chronologie ist damit weniger präzise als sie es gewesen wäre, wenn die Daten auf einem anderen Abschnitt der Kalibrationskurve lägen.

\section{Die Möglichkeit ernährungsbedingter Offsets}

Ernährungsbedingte ${ }^{14} \mathrm{C}$-Offsets können auftreten, wenn das datierte Individuum Kohlenstoff aus einem Reservoir aufgenommen hat, das nicht im Gleichgewicht mit der terrestrischen Biosphäre steht (Lanting und van der Plicht 1998). Wenn eine der Quellen dieses Reservoirs einen inhärenten Radiokarbon-Offset aufweist - wenn das datierte Individuum beispielsweise Meeresfisch oder Süßwasserfisch aus einer abgereicherten Quelle aufgenommen hat - dann wird der Knochen einen Anteil von ${ }^{14} \mathrm{C}$ aufnehmen, der nicht in Gleichgewicht mit der Atmosphäre ist. Das macht das ${ }^{14} \mathrm{C}$-Datum älter als es sein würde, wenn das betreffende Individuum eine rein auf terrestrischen Quellen basierte Nahrung zu sich genommen hätte. Werden solche Altersbestimmungen fälschlicherweise mit einer rein terrestrischen Kurve kalibriert, ergeben sie anomal frühe Datierungen (Bayliss et al. 2004).

Der Fundplatz Klingenberg „Schlossberg“ liegt über dem Neckar und das Vorkommen von Fischknochen in Befunden der MK weist darauf hin, dass die Michelsberger Bewohner Fisch zu sich nahmen (Torke 2008). In abnehmender Häufigkeit sind die am zahlreichsten vertretenen Arten: Lachs (Salmo), Barbe (Barbus barbus), Maifisch (Alosa alosa) und Döbel (Leuciscus cephalus), 
sowie nicht weiter identifizierbare Mitglieder der karpfenartigen (Cyprinidae; Torke 2008, 450_ 453). Lachs und Maifisch sind anadrome Arten, die im Süßwasser laichen und so generell marine Isotopenwerte aufweisen. Die anderen Arten leben ausschließlich in Süßwasser, was die Wahrscheinlichkeit eines Hartwasser-Offsets erhöht. Da Hunde meist Nahrung zu sich nehmen, die sie von Menschen erbeutet oder die letztere zurückgelassen haben, muss die Möglichkeit in Betracht gezogen werden, dass sich der Konsum von Fischresten auf die ${ }^{14} \mathrm{C}$-Gehalte in den Hundeknochen ausgewirkt hat. Dies ist besonders wichtig, da Hunde mehr als ein Drittel der datierten Proben stellen (Tabelle 1).

Die verlässlichste Methode, einen eventuellen Reservoir-Offset in den beprobten Hunden zu überprüfen, wären „perfekte Paare“ im Sinne von jeweils zu einem Individuum gehörigen, aneinanderpassenden Herbivoren- und Karnivoren-/Omnivorenknochen aus dem selben Kontext zu datieren (um, wie oben dargelegt, zu vermeiden, dass verlagertes Material datiert wird) und die Datierungen dann zu vergleichen. Solche perfekten Paare konnten nicht identifiziert werden. Der Idee am nächsten kommen Datierungen an verkohlten Weizenkörnern, einem verkohlten Gerstenkorn und einem fast vollständigen Hund (TS 36) aus Grube 242 (Tabelle 2: KIA-21432; MAMS-20310; SUERC-52381). Alle drei Messungen sind statistisch konsistent $\left(\mathrm{T}^{\prime}=1,8 ; \mathrm{T}^{\prime}(5 \%)=6,0 ; \nu=2\right)$, was darauf hinweist, dass aquatische Ressourcen keinen großen Anteil an der Ernährung dieses Hundes hatten. Allerdings datiert die Keramik aus dieser Grube in MK III/IV, so dass dieser Befund nicht unbedingt repräsentativ für den Großteil der Besiedlung ist. Im MK V/Munzingen Kontext 435, dem Kopf eines Segmentes des Innengrabens, sind die Datierungen am Teilskelett eines Hundes (TS 15) auf oder nahe der Grabensohle und an zwei Getreidekörnern aus der darüber liegenden Schicht ( Tabelle 2: MAMS-20320; SUERC-52401, -52406) allerdings ebenfalls statistisch konsistent $\left(\mathrm{T}^{\prime}=2,1\right.$; $\mathrm{T}^{\prime}$ $(5 \%)=6,0 ; \nu=2)$, was erneut darauf hinweist, dass aquatische Ressourcen keine signifikante Rolle bei der Ernährung dieses Hundes spielten.

FRUITS quellenproportionale Ernährungsmodelle

Trotz unseres auf die oben beschriebenen nicht wirklich perfekten „perfekten Paare“ gegründeten Priors, dass aquatische Ressourcen in Klingenberg keinen signifikanten Anteil an den von Hunden konsumierten Proteinen ausmachten, wurde versucht, die Nahrungsquellen der Klingenberger Hunde quantitativ zu rekonstruieren, wobei das bayes'sche Vermischungsmodell für stabile Isotopen FRUITS v. $2.0 \beta$ (Food Reconstruction Using Isotope Transferred Signals; Fernandes et al. 2014) zum Einsatz kam. FRUITS nutzt die isotopischen Durchschnittswerte potentieller Nahrungsquellen und erlaubt es dem Nutzer, mögliche isotopische Offsets zwischen Nahrung und Konsumenten, Gewichtung und Konzentration der Nahrungsquellen und die verfügbaren archäologischen Informationen zur wahrscheinlichen Ernährung zu definieren, um die Berechnung des Vermischungsmodells für die stabilen Isotope einzuschränken. Auf Grundlage dieser Inputs schätzt das FRUITS-Modell den durchschnittlichen Anteil (\%) und die Standardabweichung jeder Nahrungsquelle für jeden Konsumenten ab.

Die FRUITS-Modelle für Klingenberg basieren auf zwei Ernährungsproxies $\left(\delta^{13} \mathrm{C}\right.$ und $\left.\delta^{15} \mathrm{~N}\right)$.

Der isotopische Ausgangswert jeder Nahrungsquelle im Modell ist der Durchschnittswert für die 
berücksichtigten Nahrungsquellen in der betreffenden Kategorie (z.B. landlebende Pflanzenfresser; Fisch usw.; Tabelle 3) und dessen Standardabweichung. Hierfür wurden Isotopenwerte landlebender Herbivore aus Klingenberg (Rind, Rothirsch, Auerochse), dem etwa $70 \mathrm{~km}$ weiter westlich gelegenen LBK-Fundplatz Herxheim und dem mittelneolithischen Gräberfeld Trebur (Dürrwächter et al. 2006, Tabelle 1: Rothirsch, Schwein, Rind, Schaf) genutzt. Werte für Süßwasserfisch lagen nur aus Herxheim vor (Dürrwächter et al. 2006, Tabelle 1; Hecht, Karpfen). Die Durchschnittswerte dieser Nahrungsquellen und die sich aus den Standardabweichungen ergebenden Fehler dieser Durchschnittswerte wurden für unser proportionales FRUITS-Ernährungsmodell genutzt und sind in Tabelle 3 aufgelistet.

Die isotopischen Durchschnittswerte der Nahrungsquellen (Tabelle 3) deuten zwischen terrestrischen Herbivoren und Fischen einen leichten Unterschied der $\delta^{13} \mathrm{C}$-Werte $(0,4 \%)$, aber einen größeren Unterschied der $\delta^{15} \mathrm{~N}$-Werte (2,3\%) an. FRUITS benötigt einen annehmbar großen isotopischen Unterschied von 1\%o oder mehr zwischen Nahrungsquellen, um die anteiligen Mengen jeder Nahrungsquelle in der Ernährung eines Individuums verlässlich zu bestimmen. Um den vernachlässigbaren isotopischen Unterschied der $\delta^{13} \mathrm{C}$-Werte zwischen den beiden Nahrungsquellen auszugleichen, wurde das Modell so eingeschränkt, dass der Stickstoffanteil der landlebenden Pflanzenfresser in den Schätzungen der Nahrungsanteile relativ zum $\delta^{15} \mathrm{~N}$-Wert der Konsumenten als höher angesetzt wurde als der von Fisch. Dieser Prior trägt der Tatsache Rechnung, dass der erhöhte $\delta^{15} \mathrm{~N}$-Wert vieler Hunde zwar auf den Konsum einer Nahrungsquelle mit erhöhtem Stickstoffanteil (wie etwa Fisch) hinweisen mag, die archäozoologischen Ergebnisse des Fundplatzes (Stephan 2008) aber darauf hindeuten, dass Proteine landlebender Herbivore überwogen.

Als nächstes wollen wir den geeignetsten Nahrung-zu-Konsument Anreicherungsfaktor für unser FRUITS-Modell auswählen. Es existieren keine publizierten Referenzwerte speziell zu isotopischen Offsets für Hunde. Statt dessen beziehen wir uns auf die trophischen Anreicherungsschätzungen von Roth und Hobson (2000) an in Gefangenschaft lebenden Füchsen, in Anlehnung an eine ähnliche Verwendung dieser Werte durch Urton und Hobson (2005) für die Ermittlung der Variabilität in der Ernährung von Grauwölfen innerhalb einer Population. Die genutzten Nahrung-zu-Konsument Anreicherungswerte sind 2,6 $\pm 0,5 \%$ für $\delta^{13} \mathrm{C}$ und 3,4 $\pm 0,5 \%$ für $\delta^{15} \mathrm{~N}$. Die Gewichtung und Konzentration der beiden Nahrungsquellen wurden auf $100 \%$ festgelegt, womit wir Fernandes et al. 2014 für lineare (non routed) Ernährungsmodelle folgen.

In Tabelle 4 finden sich die Auflistung der $\delta^{13} \mathrm{C}_{\text {- }}$ und $\delta^{15} \mathrm{~N}_{-}-$Werte für die 21 datierten Hunde aus Klingenberg und die Ergebnisse des proportionalen Ernährungsmodelles nach FRUITS. Für die doppelt vorhandenen Analysen an manchen Hundeproben wurden gewichtete Mittel errechnet (Ward und Wilson 1978) und im Ernährungsmodell der betreffenden Individuen verwendet.

Die $\delta^{13} \mathrm{C}$-Isotopenwerte der Hunde aus Klingenberg variieren relativ wenig zwischen -19,6\%o und $-21,9 \%$ bei einer Standardabweichung von 0,7\%o vom Durchschnitt. Dagegen streuen die $\delta^{15} \mathrm{~N}$-Werte zwischen einem Minimum von 7,7\% und dem Maximum von 11,1\%0 (Differenz von 3,4\%o) und weisen eine Standardabweichung von 1.0\% um den Mittelwert von 9,6\%o auf. Diese Isotopenwerte sind vergleichbar mit den Werten, die für Hunde von Mineralbodenfundorten in Deutschland durch Ewersen und Ziegler (2014, Abb. 8 und 9) 
festgestellt wurden. Eine solche Variationsbreite der ${ }^{15} \mathrm{~N}$-Werte der Klingenberger Hunde ist bemerkenswert und deutet darauf hin, dass sich die Ernährungsweise einzelner Hunde in Art und Anteil der verfügbaren Proteinquellen unterschieden hat (Abb. 6). Die Wahrscheinlichkeit, dass mit Stickstoff angereicherte Nahrungsquellen konsumiert wurden, zeigt sich in den $\delta^{15} \mathrm{~N}$ Werten zwischen 9,9\%o und 11,1\%o und in den anschließenden FRUITS-Schätzungen für die Fischanteile in der Ernährung, die für die $\delta^{15} \mathrm{~N}$-angereicherten Hunde zwischen $10 \pm 8 \%$ und $33 \pm 15 \%$ liegen.

Die Nähe des Fundplatzes zum Neckar und die über 400 Skelettelemente von verschiedenen Fischarten, die aus Kontexten der MK in Klingenberg geborgen wurden (inkl. Alosa alosa, Leusciscus cephalus, Barbus barbus, Cyprinidae und Salmo spp; Torke 2008) weisen darauf hin, dass im Neckar gefischt wurde und die Überreste der Fische den Hunden als Kommensalen höchstwahrscheinlich zur Verfügung standen. Das Auftreten der anadromen Arten Lachs und Maifisch könnte neben dem Süßwasserreservoireffekt sogar zu einem marinen ${ }^{14} \mathrm{C}$-Offset führen.

Leider sind bisher keine Schätzungen für das Süßwasserreservoir des Neckars oder Oberrheins zugänglich. Wir untersuchen den möglichen Effekt des von unserer Ernährungsanalyse suggerierten Anteils aquatischer Ressourcen auf unsere vorgeschlagene Chronologie von Klingenberg daher auf der Grundlage eines generischen Offsets von 500 \pm 100 BP. Auf der Grundlage der bisher zugänglichen Informationen ist dies wahrscheinlich ein annehmbarer Mittelwert des marinen Offsets im Nordatlantik (von wo der Lachs und der Maifisch höchstwahrscheinlich kamen) und dem örtlichen Süßwasser-Offset im Neckar (vgl. Keaveney und Reimer 2012; Bonsall et al. 2015). Wir nutzen dieses Reservoir, mit einem Offset vom atmosphärischen Kalibrationsdatensatz (Reimer et al. 2013), und die Mix_Curves Funktion in OxCal v4.2 (Bronk Ramsey 2001, abgeändert nach Jones und Nicholls 2001). Für jeden datierten Hund haben wir eine eigene Kalibrationskurve errechnet, die den aquatischen Reservoireffekt zu dem Anteil berücksichtigt, auf den die von FRUITS ermittelten Schätzwerte der Ernährung dieses individuellen Tieres hinweisen (Tabelle 4). Beispielsweise wurde MAMS-20317 (TS 24) mit einer Kurve kalibriert, die eine Komponente von 10 $\pm 8 \%$ aquatischen Ressourcen einschließt (wobei der Anteil jeder Kurve auf 0-100\% eingeschränkt ist). Die verbleibenden

Nahrungsquellen waren wohl im Gleichgewicht mit der damaligen Atmosphäre und wurden mit IntCal13 (Reimer et al. 2013) kalibriert. Die Ergebnisse dieses Mischungsmodells aus mehreren Quellen, das diese Individuen-spezifischen Kurven mit einschließt, werden unten im Abschnitt „Alternativmodelle“ beschrieben.

\section{Struktur des Modells}

Die Struktur des bevorzugten bayes'schen Modells für michelsbergzeitliche Aktivität in Klingenberg wird in Abbildung 7 dargestellt; seine Komponenten sind in Abbildungen 8 und 10-14 im Detail zu sehen. Die MK III/IV Gruben innerhalb des Erdwerkes und die Hauptphase der MK V/Munzingen Besiedlung werden als sukzessive, kontinuierliche Aktivitätsphasen angesehen, die durch ein bestimmtes zeitliches Intervall voneinander getrennt sein könnten. Die Hauptphase umfasst Aktivitäten im äußeren Grabenring, dem Innengraben, den MK V/Munzingen Gruben innerhalb und außerhalb des Erdwerkes, sowie einige wenige 
Datierungen aus Gruben zwischen den beiden Grabenringen. Es standen keine geeigneten Proben aus Gruben im Inneren der Anlage zur Verfügung, die sowohl Keramik mit MK IV und MK V/Munzingen-Elementen enthielten. Allerdings konnten drei Gruben mit MK IV und MK $\mathrm{V} /$ Munzingen-Elementen beprobt werden, die außerhalb der Grabenringe lagen.

\section{Nutzung wäbrend MK II und III/IV (Abb. 8-9)}

Es lag kein geeignetes mit MK II-Keramik vergesellschaftetes Probenmaterial vor. Nur für zwei der Kontexte mit MK III/IV war Material für Datierungen verfügbar. In Grube 242, nahe der Spitze des Geländevorsprungs, war ein Welpe (Stephan 2008, 266: Teilskelett 36; Abb. 8: SUERC-53281) gemeinsam mit einer Konzentration von Getreidekörnern (Stika 1996, 117) deponiert worden. Davon konnten zwei Proben datiert werden (Abb. 8: KLA-21432, MAMS20310). Die drei Messungen aus Grube 242 sind statistisch konsistent $\left(T^{\prime}=1,8 ; T^{\prime}(5 \%)=6,0\right.$; $\nu=2)$. Zwei artikulierende Rothirschphalangen wurden als aus $160 \mathrm{a} / 189$ stammend dokumentiert. 160a und 189 sind zwei getrennte Gruben, die etwa $40 \mathrm{~m}$ voneinander entfernt liegen (Seidel 2008a, Abb. 220). Da allerdings beide Gruben Keramik der Phase MK III/IV enthielten, wird angenommen, dass die Datierung einer der Phalangen sich auf diese Phase bezieht (Abb. 8: SUERC-55900). Zusammengenommen deuten diese Daten darauf hin, dass MK III/IV-Gruben über einen Zeitraum von 4070-3805 cal BC (95\% Wahrscheinlichkeit) verfüllt wurden, wahrscheinlich zwischen 3985-3860 cal BC (68\% Wabrscheinlichkeit, Abb. 8: Beginn MK III/IV Gruben) und 3935-3780 cal BC (95\% Wahrscheinlichkeit), wahrscheinlich 3865-3790 cal BC (68\% W abrscheinlichkeit, Abb. 8: Ende MK III/IV Gruben), also insgesamt 1-120 Jahre (95\% Wabrscheinlichkeit), wahrscheinlich 1-70 Jahre (68\% Wabrscheinlichkeit, Abb. 17: Laufzeit MK III/IV Gruben). Diese Verteilung ist zugunsten einer kürzeren denn einer längeren Laufzeit verschoben, was darauf hinweist, dass der lange Ausläufer der Kurve, genauso wie die Begrenzungen dieser Aktivitätsphase, wegen der geringen Anzahl vorhandener Proben und dem Plateau der Kalibrationskurve im 39. Jahrhundert cal BC ungenau ist (Abb. 5).

Zusätzlich zu diesen beiden Gruben könnten auch acht weitere Daten aus MK V/Munzingen Kontexten von der gleichen Aktivitätsphase stammen. Drei Getreideproben, eine aus $264 \mathrm{im}$ Außengraben (Abb. 10: SUERC-52416), eine aus 169 im Innengraben (Abb. 11: SUERC-52410) und eine aus Grube 256 außerhalb des Erdwerks (Abb. 13: SUERC-52387) waren klar verlagert, da sie älter sind als andere Proben aus den selben Kontexten. Die verbleibenden fünf Proben bestehen aus einzelnen, nicht artikulierten Tierknochen aus Gruben, aus denen keine Vergleichsproben vorliegen (Abb. 12: MAMS-20322; Abb. 13: KLA-22467, -22468, -22469; Abb. 14: MAMS-20308). Sie kamen allerdings aus Gruben, die MK V/Munzingen Keramik enthielten (Gruben 93 (Seidel 2008b, 139-40); 484 (Seidel 2008b, 249-50, Taf. 136); 494 (Seidel 2008b, Taf. 139, 140: 3-7); 513 (Seidel 2008b, 264); und 672 (Seidel 2008b, 308, Taf. 161)). Damit sind sie definitiv älter als alle Daten aus zusammengehörenden Knochen aus Kontexten mit MK V/Munzingen Material und sind somit höchstwahrscheinlich verlagert.

Diese Daten wurden als termini post quos für ihre Kontexte in unser hier bevorzugtes Modell aufgenommen. Werden sie stattdessen als Teil einer Aktivitätsphase in MK III/IV modelliert, die auf eine Zeit vor dem Beginn der Besiedlung MK V/Munzingen festgelegt ist, so weist dieses 
separate Modell insgesamt eine gute Übereinstimmung auf (Amodel: 68) und Beginn, Ende und Laufzeit sind vergleichbar mit denen der MK III/IV Gruben allein (Beginn MK III/IV 39853825 cal BC (95\% Wabrscheinlichkeit), wahrscheinlich 3960-3880 cal BC (68\% Wabrscheinlichkeit); Ende MK III/IV 3925-3765 cal BC (95\% Wabrscheinlichkeit), wahrscheinlich 3865-3790 cal BC (68\% Wabrscheinlichkeit); Laufzeit MK III/IV 10-160 Jahre (95\% Wabrscheinlichkeit), wahrscheinlich 35-115 cal BC (68\% Wabrscheinlichkeit); Abb. 9, und weitere, hier nicht abgebildete Verteilungen-. Sollten die fraglichen Proben aus der MK III/IV Nutzungsphase des Fundplatzes stammen, dann würden die Datierungen aus Gruben 484, 494 und 672 außerhalb des Erdwerkes darauf hinweisen, dass sich diese vor-erdwerkzeitlichen Aktivitäten weit über die Verbreitung der MK III/IV-Keramik hinaus erstreckten. Bislang war die vor-erdwerkzeitliche Besiedelung auf Grundlage der Keramik als auf die Spitze des Geländevorsprunges beschränkt angesehen worden (Seidel 2008a, 286-7, Abb. 220).

\section{Nut₹ung während MK V/Mun₹ingen}

Nach einer Unterbrechung von -5 bis +135 Jahren (95\% Wabrscheinlichkeit), wahrscheinlich -5 bis +70 Jahre (68\% Wabrscheinlichkeit, Abb. 18: MK III/IV $-M K V / M z)$ begannen auf dem Bergsporn weitaus umfangreichere Aktivitäten ab 3815-3745 cal BC (95\% Wabrscheinlichkeit), wahrscheinlich 3805-3780 cal BC (68\% Wabrscheinlichkeit, Abb. 7: Beginn MKV/Mz Erdwerk. und Gruben).

\section{Der äußere Graben des Erdwerks (Abb. 10)}

Die Verfüllstruktur beider Gräben ist im Prinzip dreigeteilt. Auf der Grabensohle wurde in den meisten Grabenabschnitten eine dünne Tonschicht dokumentiert, die sich wohl in stehendem Wasser bildete (Seidel 2008a, 190-4, 225-6, Abb. 180). Über dieser Tonschicht (wo sie vorlag, sonst direkt auf der Grabensohle) lag ein 0,1-0,5 m mächtiges Paket aus feinen Straten aus Löss und von der Oberfläche eingeflossenem humosem Material. Dies kann die Folge schwerer Regenfälle gewesen sein, wobei sich jedes Jahr mehrere solcher Schichten bilden konnten (Seidel 2008a, Abb. 176). Die erhaltenen sieben bis zehn Straten dürften innerhalb weniger, vielleicht fünf Jahre nach dem Ausheben der Gräben entstanden sein (Seidel 2008a, 225f.). Über dem Sohlpaket lag ein Eintrag aus Siedlungsabfall in unterschiedlicher Dichte, darüber vermischt mit Abfall Reste von brennend in die Gräben gestürzten Holzverbauungen. Im äußeren Graben fanden sich Holzreste nur im Bereich von Grabenstück 165 und - im Gegensatz zum inneren Graben - über den Holzresten kein Wallmaterial. Die Verfüllung zeigte sich im Querprofil zudem symmetrisch (Seidel 2008a, Abb. 133-42). Es ist daher anzunehmen, dass sich in der ca. $14 \mathrm{~m}$ breiten Zone zwischen den beiden Gräben kein Wall befand. Die verbleibende Verfüllung war bis zu einem antiken Bodenbildungshorizont homogen lehmig, was auf langsame VerfüllProzesse schließen lässt. Es liegen 15 effektive Likelihoods aus fünf Graben-Abschnitten vor.

Im nördlichsten Grabenstück 13-264-71 (Seidel 2008a, 194-5); konnte eine Abfolge paläobotanischer Proben aus übereinanderliegenden Schichten im Mittelteil 264 des | Grabenstücks; gemessen werden (Stika 1996, 105, 121-3, Abb. 92-3). Vier Proben von 
verkohlten Getreidekörnern aus drei der tieferen Schichten dieser Abfolge (Abb. 10: MAMS20327, -20328, -20329; SUERC-52415) und zwei weitere, eine davon Spreu, aus einem Kontext, der nicht genau mit der ersten Abfolge in Verbindung gebracht werden konnte (Abb. 10: KL A21427,-21429), erbrachten sechs statistisch konsistente Messungen $\left(T^{\prime}=1,7\right.$; $\left.T^{\prime}(5 \%) 11,1 ; \nu=5\right)$. Eine siebte Probe ist älter und wird als terminus post quem für ihren Kontext in das Modell einbezogen (Abb. 10: SUERC-52416). Eine Messung an Eichenholzkohle aus einer verbrannten Struktur aus dem gleichen Kontext wie MAMS-20329 (Abb. 10: KIA-21428) wird als mit ihrem Kontext gleichzeitig modelliert, weil sie statistisch konsistent mit den sechs Getreideproben ist $\left(T^{\prime}=1,9 ; T^{\prime}(5 \%)=12,6 ; \nu=6\right)$. Ein im Vergleich mit den anderen Abschnitten späterer Beginn der Datierungen für 264 (Abb. 10) spiegelt die Tatsache wider, dass die Abfolge von Getreidekörnern hier erst etwa 0,40 m über der Grabensohle begann (Stika 1996, Abb. 92: i), während die meisten Proben aus den anderen Segmenten von der Sohle oder nur knapp darüber stammen (Tabelle 2).

In Grabenstück 74-212 wurden in Bereich 212, wohl nahe der Grabensohle, die beiden Vorderextremitäten eines Auerochsen gefunden; sie waren teilweise zerlegt worden und zeigten Bratspuren (Teilskelett 27; Stephan 2008, 191). Die Tatsache, dass hier mehrere zum Teil artikulierende Knochen so wie Humerus, Radius und Ulna als auch ein Metacarpus nahe beeinander liegen, weist darauf hin, dass das Tier nicht lange vor der Deponierung der Knochen zerteilt worden war, da die Reste andernfalls nicht so beeinander gefunden worden wären. Eine Probe von einem nicht der Hitze ausgesetzten Knochen wurde datiert (Abb. 10: SUERC-52419).

Im Grabenstück 214-165-194 erbrachten der Schädel und Unterkiefer eines Hundes, der knapp über der Sohle im Mittelteil 165 dokumentiert wurde (Teilskelett 37; Stephan 2008, 188, 268), zwei statistisch konsistente Radiokarbondaten (Tabelle 2: MAMS-20332.1.1, -20332.1.2) und eine dritte Messung, die nicht mit den ersten beiden konsistent ist (Tabelle 2: SUERC-52420). Ihr gewichtetes Mittel wird aus den oben beschriebenen Gründen in das Modell mit einbezogen (Abb. 10: TS 37) und hat eine gute individuelle Übereinstimmung mit seiner Position im Modell (A: 102). Im südlichen Grabenkopf 194 wurden in unterschiedlichen Höhen die Überreste zweier Hunde gefunden. Kopf, Unterkiefer und zwei Metacarpi eines Individuums (Teilskelett 38, Stephan 2008, 188, 268) wurden zwischen $1 \mathrm{~m}$ und $2 \mathrm{~m}$ unter der Oberfläche gefunden; der Schädel, Unterkiefer und zahlreiche weitere Knochen eines zweiten (Teilskelett 17; Stephan 2008, 191, 268) lagen zwischen $0 \mathrm{~m}$ und $1 \mathrm{~m}$ unter der Oberfläche. Beide Hunde erbrachten statistisch konsistente Reihen von Kontrollmessungen (Tabelle 2). Allerdings erscheint der weiter unten liegende Hund (Abb. 10: TS 38) jünger als der weiter oben liegende (Abb. 10: TS 17) und wenn beide als aufeinanderfolgend modelliert werden, wird der Übereinstimmungsindex des Modells insgesamt sehr gering (Amodel: 48). Im Grabenbereich 194 konnte keine Nachgrabung bzw. kein Grabenumbau erkannt werden. Teilskelett 38 wird daher aus dem Modell ausgeschlossen, da es klar unvollständig ist und daher möglicherweise nicht in situ liegt.

In Abschnitt 383-384 erbrachten artikulierte bzw. aneinanderpassende Rothirschwirbel (Teilskelett 16; Stephan 2008, 193), die auf oder knapp über der Sohle von 384 lagen, statistisch inkonsistente Kontrollmessungen (Tabelle 2: MAMS-20330, SUERC-52418). Ihr gewichtetes Mittel wird aus den oben beschriebenen Gründen in das Modell aufgenommen (Abb. 10: TS 16) und weist eine gute individuelle Übereinstimmung mit seiner Position im Modell auf (A:99). 
Aus dem Grabensegment 425-426-427-428-429 wurde ein größtenteils erhaltenes Hundeskelett (Teilskelett 39; Stephan 2008, 191, 268) von der Sohle des südlichen Grabenkopfes 429 datiert (Abb. 11: SUERC-52421). Sein stratigraphisches Verhältnis zu einem unvollständigeren Hund aus demselben Grabenkopf (Teilskelett 44; Stephan 2008, 191, 268) ist unbekannt (Abb. 10 : MAMS-20334). Ein Datum der untersten Schicht von Grabenkopf 425 wird als gleichzeitig mit seinem Kontext angesehen, weil es eine gute Übereinstimmung aufweist, wenn es als gleichzeitig mit den Datierungen für die beiden Hunde modelliert wird (Abb. 10: Hd-15523,-16282).

Auf dieser Grundlage wurde der äußere Graben 3810-3735 cal BC (95\% Wabrscheinlichkeit), wahrscheinlich 3805-3775 cal BC (68\% Wabrscheinlichkeit, Abb. 10: Bau Außengraben-) ausgehoben, und 3675-3640 cal BC (95\% Wabrscheinlichkeit), wahrscheinlich 3665-3645 cal BC (68\%

Wabrscheinlichkeit, Abb. 10: Auflassen Außengraben), nach einem Intervall von 85-160 Jahren (95\% Wahrscheinlichkeit), wahrscheinlich 120-150 Jahre (68\% Wabrscheinlichkeit, Abb.17: Nutzung Außengraben) aufgegeben.

Der innere Graben des Erdwerks (Abb. 11)

Der innere Graben weist - wie der Außengraben - -ein gebändertes Sohlpaket auf, - das namentlich an den tiefsten Stellen -eine dünne, in Wasser abgelagerte Tonschicht überdeckt. Darüber findet sich eine „Abfallschicht“, die im inneren Graben entlang des gesamten Segments 225-169-195 und in den Segmenten 022-268 und 081-219 nur im Torbereich von brennend in den Graben gestürzten Holzelementen bedeckt war. Am zentralen Durchlass 386 zu 387 fanden sich zudem Steine aus einer Trockensteinmauerkonstruktion. Unmittelbar auf das verbrannte Holz bzw. die Steine war im inneren Graben von der Innenseite her Löss einer Wallschüttung in den Graben gebrochen, der auf die Existenz eines Walls innerhalb des inneren Grabens hinweist (Abb. 3). Er dürfte innerhalb eines $7 \mathrm{~m}$ entfernten Palisadengräbchens platziert gewesen sein (Seidel 2008a, 201-17). Unter diesen Umständen erschien es lohnenswert, Proben aus Befunden zu untersuchen, die unter dem Wall gelegen haben müssen und daher termini post quos für dessen Aufschüttung geben könnten. Dies erwies sich allerdings als schwer umsetzbar, insbesondere weil Volumen, Breite und genaue Verortung des Walles nicht abschließend geklärt sind (Seidel 2008a, 221-9; Jacob 2010, 42-9). Es konnte daher nicht mit Sicherheit festgestellt werden, welche Befunde im Bereich bzw. unter dem Wall lagen. Angenommen wurde, dass Grube 171, die $15 \mathrm{~m}$ von der gegenwärtigen Innenkante des Grabens entfernt ist, vom Wall überlagert wurde (Seidel 2008a, 223). Allerdings erbrachte ein Einzelknochen aus diesem Befund einen terminus post quem von 3720-3640 cal BC (92\% Wabrscheinlichkeit), wahrscheinlich 3700-3655 (68\%

Wabrscheinlichkeit, Abb. 12: Hd-15522 -16384), der später datiert als Proben, die aus dem inneren Graben von der Sohle oder knapp darüber stammen. Nimmt man diesen Knochen als ein Datum vor dem Ausheben des Grabens in das Modell auf, ergibt sich eine insgesamt schlechte Übereinstimmung (Amodel: 44). Entweder lag die Grube hinter der maximalen Breite des Walles, oder sie wurde angelegt und verfüllt, als der Graben bereits wieder eingeebnet war. Ein Tierknochen eines Teilskeletts, der in Kombination mit MK V/Munzingen Keramik in Grube 170, nahe 171 und noch näher an der Grabenkante lag (Abb. 2), erbrachte statistisch konsistente Kontrollmessungen, allerdings datieren diese in die LBK (Tabelle 2: MAMS-20323, SUERC52407). Ein Einzelknochen aus Grube 93, $10 \mathrm{~m}$ innerhalb der Grabenkante, könnte einen 
terminus post quem von 3960-3900 cal BC (28\% Wabrscheinlichkeit) oder 3885-3800 cal BC (67\% Wabrscheinlichkeit), wahrscheinlich 3950-3935 cal BC (9\% Wabrscheinlichkeit) oder 3875-3810 cal BC (59\% Wabrscheinlichkeit, Abb. 12: MAMS-20322) für das Grabenwerk erbringen, kann das Modell aber nur wenig präzisieren, weil es sich um einen einzelnen Knochen handelt und die Probe deutlich älter datierte als diejenigen von der Grabensohle. Der Knochen aus Grube 93 gehört zu den Proben, die als wahrscheinlich zur MK III/IV Besiedlung gehörig angesehen werden (Abb. 9). Diese Probe und die aus Grube 171 werden in unserem bevorzugten Modell daher lediglich als termini post quos für die Gruben verwendet, aus denen sie stammen (Abb. 12). MAMS-20322 gibt auch einen terminus post quem für den außergewöhnlichen Fund mehrerer Tonbrüste, die ursprünglich eine (Haus?-)Wand zierten (Seidel 2008a, 301-8, Abb. 230-5).

Die Datierung des inneren Grabens stützt sich daher auf Proben aus dem Graben selbst. Obwohl zwei Fragmente aus der großen Menge verkohlter Hölzer, die in Teilen des Grabens dokumentiert werden konnten, bereits früher datiert worden waren, konnten im Rahmen des ToTL-Projektes keine Holzreste mehr aufgefunden werden. Für ergänzende Proben wurde daher auf verkohlte Pflanzenreste und Teilskelette zurückgegriffen. Es liegen 14 effektive Likelihoods, darunter drei Sätze von Kontrollmessungen, aus drei Abschnitten vor.

In Bereich 169, dem mittleren Teil von Grabenabschnitt 225-169-195, enthielt das gebänderte Sohlpaket wenig Artefakte und erbrachte keine geeigneten Proben. Die „Abfallschicht“ war durch zahlreiche verbrannte Hölzer überlagert, die teilweise mit ihr vermischt waren (Abb. 3: Seidel 2008a, 206-12). Die Eichenteile der verstürzten Verbauung dürften kurz vor ihrer Verbauung gefällt worden sein. Allerdings konnten keine Proben mehr aufgefunden werden, die die beiden vorhandenen Datierungen hätten ergänzen können. Letztere kamen von einem Brett und einer Stange von ca. 0,15 m Durchmesser. In keinem Fall wurde dokumentiert, ob es sich bei den Proben um Kernholz, Splintholz oder beides handelte, so dass, wegen der Langlebigkeit der Eiche, beide als termini post quos für den Bau der Palisade modelliert werden müssten. Allerdings ist die Messung an dem Brett statistisch konsistent mit vier Messungen an verkohlten Getreideresten aus der „Abfallschicht“ ( $\left.\mathrm{T}^{\prime}=1,8 ; \mathrm{T}^{\prime}(5 \%)=9,5 ; \nu=4\right)$. Es wird daher als gleichzeitig mit ihrem Kontext modelliert (Abb. 11: KIA-21425). Die Datierung der Stange fällt wie erwartet jünger aus, da sie aus einem dünneren Rundholz bestand (Abb. 11: KIA-21426).

Die zahlreichen verkohlten Pflanzenreste aus 169 waren zwischen und um die verkohlten Bauhölzer konzentriert (Stika 1996, 106-8, 123-5, Abb. 95-7). Sechs Proben an Erbsen und Getreide fielen ins späte sechste bis frühe fünfte Jahrtausend cal BC und gehören wohl zur bandkeramischen Besiedlung des Platzes (Tabelle 2: KIA-21421, -21422, -21424, -21436;

MAMS-20325; SUERC-52409). Weitere vier Proben an Getreidekörnern ergaben statistisch konsistente Messungen $\left(\mathrm{T}^{\prime}=1,0 ; \mathrm{T}^{\prime}(5 \%)=7,8 ; \nu=3\right.$; Abb. 11: KIA-21423; MAMS-20324, -20326; SUERC-52408). Eine fünfte ist älter und fällt ins 40. bis 39. Jahrhundert cal BC; das Korn war wohl verlagert und wird als terminus post quem modelliert (Abb. 11: SUERC-52410).

169-195-225 war der südlichste Grabenabschnitt mit verkohlten Hölzern. In den beiden südlich anschließenden Abschnitten, 197-386 und 387-434, wurde am zentralen Durchlass 386 zu 387 die entsprechende stratigraphische Position von flachen Kalksteinplatten eingenommen. Sie waren wohl aus einem Trockenmauerwerk aus Bruchsteinen gefallen (Seidel 2008a, 213-14, 225; Abb. 177). Die verbleibenden zwei Grabenabschnitte, aus denen Proben entnommen wurden, 
lagen noch weiter im Süden. Dort fanden sich keine Hinweise auf ein Brandereignis und Funde waren seltener.

Im Grabensegment 435-436-437-438-439 wurden nahe der oder auf der Sohle des nördlichen Grabenkopfs 435, der Schädel, Unterkiefer und die beiden Halswirbel eines Hundes (Teilskelett 50: Stephan 2008, 188, 268) freigelegt (Abb. 11: SUERC-52406). Sie lagen unter einer Schicht mit verkohlten Pflanzenresten, die zwar seltener als in 169 aber noch immer zahlreich waren (Stika 1996, 123-5). Zwei einzelne Gerstenkörner aus dieser Schicht wurden datiert (Abb. 11: MAMS-20320, SUERC-52401). Ein etwa in der Mitte des Abschnittes nicht im Verband liegender Knochen nahe der Sohle wird als gleichzeitig mit seinem Kontext angesehen, weil seine Datierung mit denen der Getreidereste und Teilskelette an anderen Stellen in diesem Abschnitt übereinstimmt (Abb. 11: Hd-15526-16352). Aus dem südlichen Grabenkopf 439 wurde ein auf oder knapp über der Sohle gut erhaltener Hund (Teilskelett 15; Stephan 2008, 268) beprobt. Es handelt sich hier um zwei statistisch inkonsistente Kontrollmessungen (Tabelle 2: MAMS-20321, SUERC-52405), deren gewichtetes Mittel aber aus den oben dargelegten Gründen in das Modell einbezogen wird und wieder eine gute individuelle Übereinstimmung mit seiner stratigraphischen Position im Modell aufweist (Abb. 11: TS 15; A: 99).

In Abschnitt 441-442 wurden zwei Hunde aufgefunden, die erst während der weiteren Bearbeitung getrennt werden konnten. Sie lagen auf oder knapp über der Sohle im nördlichen Bereich des Befundes (Teilskelette 14/1 und 14/2; Stephan 2008, 191, 268-9). Jeder erbrachte ein Paar statistisch konsistenter Kontrollmessungen (Abb. 11: TS 14/1, TS 14/2).

Auf dieser Grundlage wurde der Innengraben 3805-3740 cal BC (95\% Wabrscheinlichkeit), wahrscheinlich 3800-3770 cal BC (68\% Wabrscheinlichkeit, Abb. 11: Bau Innengraben) ausgehoben und 3680-3635 cal BC (95\% Wabrscheinlichkeit), wahrscheinlich 3665-3640 cal BC (68\% Wabrscheinlichkeit, Abb. 11: Auflassen Innengraben) nach einem Zeitraum von 80-160 Jahren (95\% Wahrscheinlichkeit), wahrscheinlich 110-150 Jahre (68\% Wahrscheinlichkeit; Abb. 17: Nutzung Innengraben) aufgelassen.

\section{Gruben der Phase MK V/Munzingen}

Insgesamt liegen 33 effektive Likelihoods für Gruben mit Material der Stufe MK V/Munzingen vor. Die Keramik aus drei Grubenumfasst auch typologische Elemente der Stufe MK IV.

MK V/Munzingen Befunde im Inneren des Erdwerks (Abb. 12). Es liegen 12 effektive Likelihoods für zehn der MK V/Munzingen Gruben innerhalb des Erdwerks vor. Die Proben bestehen aus verkohlten Getreidekörnern aus 236, 271 und 272 (Abb. 12: KIA-21431; SUERC-52385, -52386; MAMS-20311, -20313); Hundeskeletten oder Teilskeletten aus 116, 238 und 357 (Abb. 12: SUERC-52380, MAMS-20309, -20312); einem Paar Rothirschgeweih aus 305 (Abb. 12: SUERC55895); und einzelnen, nicht im Verband liegenden Tierknochen aus 93, 171 und 346 (Abb. 12: Hd-15522-16384, -15528-16337; MAMS-20322). Letztere werden als termini post quos für ihre Kontexte behandelt, da sie verlagert sein könnten. Auf dieser Grundlage begann die Verfüllung der Gruben 3800-3735 cal BC (95\% Wahrscheinlichkeit), wahrscheinlich 3795-3760 cal BC (68\% Wabrscheinlichkeit, Abb. 12: Beginn $M K V / M z$ Gruben innerbalb des Erdwerks), ein Prozess der bis 
3665-3630 cal BC (95\% Wabrscheinlichkeit), wahrscheinlich 3655-3640 cal BC (68\%

Wahrscheinlichkeit, Abb. 12: Ende MK V/Mz Gruben innerbalb des Erdwerks) andauerte, also über einen geschätzten Zeitraum von 85-160 Jahren (95\% Wabrscheinlichkeit), wahrscheinlich 115-150 Jabre (68\% Wabrscheinlichkeit, Abb. 17: Nutzung MK V/Mz Gruben innerbalb des Erdwerks).

MK V/Munzingen Befunde außerhalb des Erdwerks (Abb. 13). Es existieren 18 effektive Likelihoods, inklusive zwei Sätze Kontrollmessungen, aus 17 Befunden. Die Proben umfassen verkohlte Getreidekörner aus 256 (Abb. 13: MAMS-20314; SUERC-52387); ein Skelett eines neugeborenen Kindes aus Grube 500 (Abb. 13: MAMS-19231); neun Hundeskelette oder Teilskelette aus 408, 483, 499, 567, 616, 657, 660, 669 und 677 (Abb. 13: SUERC-52389, -52390, -52391, -52395; MAMS-20315, -20316, 20-317; TS 25, TS 40); ein vereinzeltes Fragment Eichenholzkohle aus 478 (Abb. 13: KIA-21435) und einzelne, nicht im Verband liegende Tierknochen aus 73, 484, 505 und 672 (Abb. 13: KIA-22467, -22468, -22469; Hd-15524-16283; SUERC-52417). Letztere fließen als termini post quos für ihre Kontexte in das Modell ein, da sie verlagert worden sein könnten. Die Eichenholzkohle wird ebenso behandelt, da der Baum schon alt gewesen sein könnte, als er gefällt wurde. Von zwei Hunden, Teilskelette 46 und 25 (Stephan 2008, 266-7), liegen Kontrollmessungen vor. Die Messungen für Teilskelett 46 aus Grube 677 sind statistisch konsistent (Tabelle 2: MAMS-20319; SUERC-52396). Zwei der Messungen für Teilskelett 25 aus Grube 408 sind ebenfalls statistisch konsistent (Tabelle 2: MAMS-20318.1.1, 20318.1.2), die dritte ist nicht mit ihnen statistisch konsistent (Tabelle 2: SUERC-52388). Die gewichteten Mittel für beide Hunde weisen aber trotzdem eine gute individuelle Übereinstimmung im Modell auf (Abb. 13: TS 46, TS 25).

Auf dieser Grundlage wurde 3790-3725 cal BC (95\% Wahrscheinlichkeit), wahrscheinlich 3780 3745 cal BC (68\% Wabrscheinlichkeit, Abb. 13: Beginn MK IV/V und V/Mz Gruben außerbalb des Erdwerks; Abb. 13) mit der Verfüllung der Gruben außerhalb des Erdwerks begonnen, ein Prozess der sich bis 3680-3640 cal BC (95\% Wabrscheinlichkeit), wahrscheinlich 3670-3645 cal BC (68\% Wabrscheinlichkeit, Abb. 13: Ende MK IV/V und V/Mz. Gruben außerhalb des Erdwerks), also über einen geschätzten Zeitraum von 65-135 Jahren (95\% Wabrscheinlichkeit), wahrscheinlich 90 120 Jahre (68\% Wabrscheinlichkeit, Abb. 17: Nutzung MK IV/V und V/Mz Gruben außerbalb des

Erdwerks); fortsetzte. Es ist bemerkenswert, dass die Datierungen der Hundeskelette aus drei Gruben, die neben MK V/Munzingen Elementen auch MK IV Keramik enthalten (616, 669 und 677; Seidel 2008b, Taf. 159: 1, Taf. 161: 5-6; Taf. 161: 4), später als MK III/IV datieren, also in den Zeitraum der MK V/Munzingen Keramik fallen (Abb. 13: SUERC-52391, -52393; TS 46).

MK V/Munzingen Befunde zwischen den Erdwerksgräben (Abb. 14). Drei Daten stammen aus Gruben, die zwischen den Erdwerksgräben liegen. Es ist unwahrscheinlich, dass die Gruben ausgegraben und genutzt wurden, während die Gräben bestanden. Sie könnten daher termini post quos oder ante quos für das Erdwerk liefern. Eine Datierung, MAMS-20308 aus Grube 513, ist klar älter als das Erdwerk. Zwei fallen allerdings in den Nutzungszeitraum der Gräben (Abb. 14: KIA-21430; SUERC-52379). Jedoch könnten diese aus späteren Befunden stammen, die aus der Nutzungsphase des Erdwerks verbliebenes Material enthielten, da es sich bei den Proben um ein einzelnes Getreidekorn von insgesamt vieren aus Grube 77 und um einen Einzelknochen aus Grube 385 handelte. Es bleibt unklar, ob Gruben zwischen den Gräben auch während der Nutzungsphase des Erdwerks angelegt wurden bzw. offen standen. 


\section{Hundedeponierungen}

Abbildung 15 zeigt die von unserem bevorzugten Modell (Abb. 7-8 und 10-14) errechneten Datierungen der Überreste von Hunden aus MK V/Munzingen Zusammenhängen und schätzt den Beginn, das Ende und die Dauer der Hundedeponierungen auf diesem Fundplatz in dieser Periode ein. TS 38 aus Profil 194 im Außengraben wird dabei nicht berücksichtigt, weil es aus den oben genannten Gründen aus dem Modell ausgeschlossen wurde. Die Hundedeponierungen der Phase MK V/Munzingen wurden zwischen 3810-3740 cal BC (95\% Wabrscheinlichkeit), wahrscheinlich 3805-3775 cal BC (68\% Wabrscheinlichkeit, Abb.15: Beginn MKV/Mz Hunde) und 3665-3635 cal BC (95\% Wahrscheinlichkeit), wahrscheinlich 3655-3640 cal BC (68\%

Wabrscheinlichkeit, Abb. 15: Ende $M K V / M z$ Hunde;), also über einen Zeitraum von 95-165 Jabren (95\% Wabrscheinlichkeit), wahrscheinlich 130-155 Jabre (68\% Wabrscheinlichkeit, Abb. 17: Laufzeit $M K V / M z$ Hunde) angelegt. Sie erstrecken sich also über den gesamten MK V/Munzingen Nutzungszeitraum (Abb. 18) und sind in allen während dieser Zeit genutzten Befundarten vertreten. Einen früheren Beginn für die Hundedeponierungen belegt das Datum 3960-3890 (37\% Wabrscheinlichkeit) oder 3885-3805 cal BC (58\% Wabrscheinlichkeit), wahrscheinlich 39553925 cal BC (18\% Wahrscheinlichkeit) oder 3880-3815 cal BC (50\% Wabrscheinlichkeit), das an einem Welpen aus Grube 242 gemessen wurde, die Keramik der Stufe MK III/MK IV enthielt (Abb. 8: SUERC-52381).

\section{Synthese}

Die Schlüsselparameter des bevorzugten Modells sind in Abb. 16-18 zusammengefasst und in den Tabellen 5 und 6 aufgelistet. Tabelle 7 zeigt die Abfolge der wichtigsten Ereignisse in der Geschichte des Fundplatzes.

Nutzung vor $M K V / M u n z i n g e n$

Die Nutzung während MK II ist mit typologischen Argumenten vor der Besiedlung während MK III/IV anzusetzen. Letztere beginnt 4070-3805 cal BC (95\% Wahrscheinlichkeit), wahrscheinlich 3985-3860 cal BC (68\% Wahrscheinlichkeit, Abb. 16: Beginn MK III/IV Gruben). Die Aufgabe der MK III/IV Gruben erfolgte dann 3935-3780 cal BC (95\% Wahrscheinlichkeit), wahrscheinlich 3865-3790 cal BC (68\% Wabrscheinlichkeit, Abb. 16: Ende MK III/IV Gruben), nach 1-120 Jahren (95\% Wabrscheinlichkeit), wahrscheinlich nach 1-70 Jahren (68\% Wabrscheinlichkeit, Abb. 17: Laufzeit MK III/IV Gruben). Daraufhin folgte eine Unterbrechung von -5 bis +135 Jabren (95\% Wabrscheinlichkeit), wahrscheinlich -5 bis +70 Jahre (68\% Wabrscheinlichkeit, Abb. 18: $M K I I I / I V-M K V / M z)$ bis zum Beginn der Besiedlung während MK V/Munzingen. Werden zusätzlich Datierungen von Proben, die wahrscheinlich oder sicher sekundär in jüngere Befunde verlagert wurden, in die Nutzungsphase MK III/IV miteinbezogen (Abb. 9), so ändern sich die Schätzwerte nur leicht; ihre Mediane variieren um nur 14 Jahre. 
Die Nutzung während MK V/Munzingen begann wahrscheinlich mit dem Bau des Erdwerks (92 \% Wabrscheinlichkeit). Der äußere Graben wurde 3810-3735 cal BC (95\% Wabrscheinlichkeit), wahrscheinlich 3805-3775 cal BC (68\% Wabrscheinlichkeit, Abb. 16: Bau Außengraben) ausgehoben. Es ist zu 71\% wabrscheinlich, dass der äußere Graben vor dem inneren Graben angelegt wurde (Tabelle 7). Letzterer wurde 3805-3740 cal BC (95\% Wabrscheinlichkeit), wahrscheinlich 38003770 cal BC (68\% Wabrscheinlichkeit, Abb. 16: Bau Innengraben) ausgehoben. Wird die Differenz aus diesen beiden Schätzungen ermittelt, kann abgeschätzt werden, wie viele Jahre zwischen dem Ausheben der beiden Gräben vergingen (Abb. 18, Tabelle 6). Der resultierende Schätzwert ist teilweise negativ, weil die beiden Wahrscheinlichkeitsverteilungen überlappen. Der innere Graben wurde -15 bis +35 Jahre (95\% Wabrscheinlichkeit), wahrscheinlich -10 bis +15 Jabre (68\% Wahrscheinlichkeit) nach dem äußeren Graben angelegt. Das heißt, dass beide in einem Zug ausgehoben worden sein könnten. Wenn das nicht der Fall war, so wurden sie doch sehr schnell hintereinander angelegt, insbesondere weil der Median dieser Verteilung nur 4 Jahre beträgt (Abb. 18: Außengraben/Innengraben). Dies erlaubt auch eine erste Schätzung, wie lange der Bau der beiden Grabenzüge gedauert haben könnte.

Nachdem das Grabenwerk errichtet worden war, wurden die ersten MK V/Munzingen Gruben im Inneren der Anlage ausgehoben, und zwar ab 3800-3735 cal BC (95\% Wabrscheinlichkeit), wahrscheinlich ab 3795-3760 cal BC (68\% Wabrscheinlichkeit: Abb. 16: Beginn MK V/Mz Gruben innerbalb des Erdwerks). Nach -25 bis +55 Jahren (95\% Wabrscheinlichkeit; Abb. 18: Gruben Innenraum/Gruben Außenraum), wahrscheinlich -10 bis +30 Jabren (68\% Wabrscheinlichkeit), wurden MK V/Munzingen Gruben dann auch außerhalb der Erdwerksgräben angelegt. Gruben der Phasen MK IV/V und MK V/Munzingen standen hier wohl ab 3790-3725 cal BC (95\% Wabrscheinlichkeit), wahrscheinlich 3780-3745 cal BC (68\% Wabrscheinlichkeit; Abb. 16: Beginn MK $V / M_{z}$ Gruben außerbalb des Erdwerks) offen.

Wird die Abfolge der Anfangszeitpunkte dieser Ereignisse (Tabelle 7) näher beleuchtet, scheint | es, als ob das Erdwerk und die zugehörigen Gruben nicht alle zu genau der-selben Zeit begannen, sondern dass sich hier eine gewisse Entwicklungsdauer andeutet. Diese war allerdings relativ kurz. Beispielsweise liegt der zeitliche Abstand zwischen den wahrscheinlich ältesten und jüngsten Elementen (also dem Außengraben und den Gruben außerhalb des Erdwerks) bei -10 bis +65 Jahren (95\% Wabrscheinlichkeit), wahrscheinlich +5 bis +40 Jahre (68\% Wabrscheinlichkeit, Abb. 18: Außengraben/ Gruben außen). Die gesamte Anlage entstand also wahrscheinlich im Laufe einer Generation.

Die datierten Proben decken nicht die gesamte Grabentiefe ab, sondern stammen vor allem aus den fundreichen Ablagerungen in den unteren Schichten, die sich auf die neolithische Nutzung des Erdwerks beziehen. Dem Modell zufolge endete die Nutzung des äußeren Grabens 3675 3640 cal BC (95\% Wabrscheinlichkeit), wahrscheinlich 3665-3645 cal BC (68\% Wabrscheinlichkeit, Abb. 16: Auflassen Außengraben). Die Nutzung des Innengrabens endete 3680-3635 cal BC (95\% Wabrscheinlichkeit), wahrscheinlich 3665-3640 cal BC (68\% Wabrscheinlichkeit, Abb. 16: Auflassen Innengraben). Vergleichen wir die Differenz zwischen diesen beiden Verteilungen, so scheint es, dass beide Gräben genau zur gleichen Zeit aufgelassen wurden.

Gruben im Außenbereich wurden ab 3680-3640 cal BC (95\% Wabrscheinlichkeit), wahrscheinlich 3670-3645 cal BC (68\% Wahrscheinlichkeit, Abb.16: Ende MK V/Mz Gruben außerhalb des Erdwerks) 
nicht mehr angelegt und verfüllt. Zeitlich liegt dies sehr nah am Auflassen der Gräben selbst. Wenn diese Ereignisse also nicht absolut gleichzeitig waren, dann lagen sie nur wenige Jahre auseinander. Gruben im Innenraum des Erdwerks könnten noch ein wenig später genutzt worden sein (Tabelle 7). In Innenbereich endete das Ausheben von Gruben 3665-3630 cal BC (95\% Wabrscheinlichkeit), wahrscheinlich 3655-3640 cal BC (68\% Wabrscheinlichkeit, Abb. 16: Ende MK V/Mz Gruben innerbalb des Erdwerks). Dies geht allerdings auf nur eine Messung zurück, einen Hund aus Grube 238 (Abb. 12: MAMS-20309), der bis zu einem Jahrzehnt nach der Zerstörung des Erdwerks deponiert worden sein könnte.

All dies könnte auf ein einziges Ereignis hinweisen, im Laufe dessen der Brand der Wall-GrabenAnlage zur Zerstörung des Erdwerks und der Aufgabe des Platzes führte. Die Tatsache, dass die Verfüllschichten oberhalb des Brandereignisses fast steril sind, deutet ebenfalls auf ein de facto Ende der Besiedlung nach der Zerstörung des Erdwerkes hin. Die Hundedeponierung in Grube 238 könnte daher ein kleiner, nachträglicher Akt des Gedenkens gewesen sein, sofern sie nicht in den Jahren unmittelbar vor oder am Ende des Erdwerks niedergelegt wurde.

Insgesamt dauerte die MK V/Munzingen Nutzungsphase des Platzes 95-170 Jahre (95\% Wabrscheinlichkeit) wahrscheinlich 135-160 Jabre (68\% Wabrscheinlichkeit, Abb. 17: Laufzeit MK $V / M z$ Erdwerk, und Gruben). Der Außengraben wurde 85-160 Jabre (95\% Wabrscheinlichkeit) wahrscheinlich 120-150 Jahre (68\% Wahrscheinlichkeit; Abb. 17: Nutzung Außengraben) genutzt, der Innengraben über eine ähnliche Zeitspanne (Tabelle 6; Abb. 17).

\section{Alternativmodelle}

Das oben beschriebene bevorzugte Modell ist keineswegs die einzige Möglichkeit, die Chronologie des Fundplatzes zu interpretieren. Weitere Möglichkeiten werden im Folgenden kurz beschrieben, um festzustellen, ob die Abänderung einiger Aspekte der a-prioriInformationen wesentliche Auswirkungen auf die hier vorgeschlagene Chronologie hätte.

\section{Alternative 1: Was, wenn einige der MK V/Munzingen Gruben älter sind als das Grabenwerk?}

Das hier bevorzugte Modell beinhaltet keine Angaben zum heute verschwundenen Wall des Erdwerks und den Befunden, die unter ihm gelegen haben könnten, weil die Lage und die Breite des Walles nicht eindeutig bestimmt werden können. Obwohl das Ausheben der Gruben im Innenraum wohl erst nach dem Bau des Erdwerkes einsetzte, bleibt es nach wie vor möglich, dass einige dieser Gruben früher datieren (Abb. 18: Außengraben/ Gruben Innenraum, Innengraben/ Gruben Innenraum). Wird die Laufzeit beider Gräben im Modell so eingeschränkt, dass sie nach dem Beginn der MK V/Mz Gruben im Innenraum datieren, hat das Modell insgesamt eine gute Übereinstimmung (Amodel: 72), auch wenn das mögliche Intervall zwischen dem Beginn der Gruben im Inneren und dem Ausheben der Gräben nur gering ist, für den Außengraben beträgt es -5 bis +20 Jabre (95\% Wabrscheinlichkeit), wahrscheinlich -5 bis +10 Jahre $(68 \%$

Wabrscheinlichkeit, Verteilung nicht abgebildet). 


\section{Alternative 2: Könnten einige MK V/Munzingen Gruben später datieren als das Erdwerk?}

Unserem bevorzugten Modell nach wurden MK V/Mz Gruben im Innenbereich noch eine kurze Zeit nach dem Auflassen der Gräben ausgehoben und verfüllt, höchstens noch 30 Jahre, aber wahrscheinlich nicht mehr als ein Jahrzehnt lang (Abb. 18, Tabelle 6: Ende Außengraben / Ende Gruben Innenraum). Dies beruht auf der Datierung des Hundeskelettes aus Grube 238. Es gibt zudem Argumente, die dafür sprechen dass einige weitere Gruben später datieren könnten als das Erdwerk. Gruben 236 und 346, beide im Innenbereich der Anlage, könnten Material enthalten, dass zeitgleich mit dem Niederbrennen der Palisade ist (Seidel 2008a, 248-9). Sie könnten daher nach dem Brandereignis verfüllt worden sein; aus 346 wurde typologisch späte Keramik geborgen, darunter ein flachbodiger Tulpenbecher und eine Tasse (Seidel 2008b, Taf. 120, 121). Zwei andere Befunde könnten aus räumlichen und chronologischen Gründen nach der Zerstörung des Erdwerks verfüllt worden sein. Die Messung aus Grube 171, die vielleicht im Bereich unter dem ehemaligen Wall liegt, ist — wie bereits erläutert — zu jung um älter als die Wallaufschüttung zu datieren. Sie könnte daher später genutzt worden sein. Grube 73 war wohl kaum zeitgleich mit dem Außengraben, da sie genau in der Mitte des Grabensystems und knapp vor einer Erdbrücke lag, am Durchlass zwischen 74 und 71 (Abb. 2). Die Datierung ist hier ebenfalls zu jung als dass die Grube vor dem Bau des Erdwerkes angelegt worden sein könnte. Die Datierungen aus den Gruben 73, 171 und 346 sind termini post quos, da sie an einzelnen Tierknochen gemessen wurden (Abb. 14: SUERC-52417; Abb. 13: Hd-15522-16384, -1552816337). Diese -Gruben könnten also durchaus später datieren. Nur die Getreideproben aus Grube 236 und das Hundeskelett aus Grube 238 werden im bevorzugten Modell als gleichzeitig mit ihren Kontexten angesehen (Abb. 12: KIA-21431, MAMS-20309). Werden alle fünf diskutierten Gruben als nach der Aufgabe des Erdwerkes fallend modelliert, weist das Modell noch immer eine gute Übereinstimmung auf (Amodel: 92). Die Zeitspanne zwischen dem Auflassen des Außengrabens und dem Verfüllen der Gruben wird auf -5 bis +30 Jabre (95\% Wabrscheinlichkeit), wahrscheinlich +5 bis +20 Jabre (68\% Wabrscheinlichkeit, Verteilung nicht abgebildet) geschätzt. Mit anderen Worten, selbst wenn weitere Gruben nach dem Fall der Grabenanlage datiert würden, würde die Zeitspanne der Nutzung insgesamt gleich bleiben das Ausheben der Gruben scheint innerhalb etwa eines Jahrzehnts aufgehört zu haben, wie auch im bevorzugten Modell (Abb. 18: Ende Außengraben / Ende Gruben Innenraum; Ende Innengraben / Ende Gruben Innenraum).

\section{Alternative 3: Was, wenn einige Datierungen signifikant von Ernährungs-Offsets beeinflusst worden wären?}

Die letzte Empfindlichkeitsanalyse untersucht die Möglichkeit, dass Süßwasser- und anadrome Fische in der Ernährung der Hunde von Klingenberg einen Reservoireffekt auf deren Radiokarbondaten ausgeübt haben. Das in Abbildungen 7-8 und 10-14 definierte Modell wurde noch einmal mit IntCal13 nachgerechnet, wobei eine voll terrestrische Nahrung und eine individuelle Kalibrationskurve für jeden Hund zu Grunde gelegt wurden. Die Kurve beinhaltet ein Reservoir-Offset von 500 100 BP in dem Verhältnis, wie es die mittels des FRUITS-Modells vorgeschlagene Ernährung für das betreffende Tier nahelegt (Tabelle 4). 
Dieses Modell hat eine gute Übereinstimmung (Amodel: 77; Modell nicht abgebildet). Insgesamt verschieben sich die Enddaten der Hauptereignisse aus Tabelle 5 nur um einige Jahre (obwohl das Ende der MK III/IV Gruben bis zu eine Generation später stattgefunden haben könnte).

| Die vorgeschlagenen Anfangszeitpunkte für die-selben Ereignisse reagieren empfindlicher auf dieses Alternativmodell und datieren jeweils etwa ein halbes Jahrhundert später.

Dementsprechend reduziert sich auch die Länge dieser Aktivitätsperioden um 40-50 Jahre.

Wenn Teilskelett 38, das aus dem bevorzugten Modell ausgeschlossen worden war, weil es dort

| eine schlechte Übereinstimmung zur Folge hatte, in das Mischkurvenmodell mit einbezogen wird, so hat auch dieses Modell insgesamt eine gute Übereinstimmung (Amodel: 72) und das stratigraphische Verhältnis zwischen den beiden Hunden (Teilskelette 17 und 38) in Abschnitt 214-165-194 im Außengraben ist nicht mehr problematisch.

Innerhalb der Genauigkeitsspanne der modellierten Chronologie für Klingenberg könnten solche Offsets für die weitere Interpretation wichtig sein (vor allem für ein dendrochronologisches Zeitgerüst). Wir bleiben jedoch skeptisch, was die Genauigkeit dieses Modells angeht. Zunächst liefern die beiden bereits beschriebenen nicht perfekten „,perfekten Paare“ keinen Hinweis für einen Reservoireffekt im Alter der beiden Hunde TS 15 (mit einem geschätzten Süßwasserfischkonsum von 4 $\pm 4 \%$ ) und TS 36 (mit einem geschätzten Süßwasserfischkonsum von $13 \pm 10 \%)$. Obwohl die Variationsbreite in den $\delta^{15} \mathrm{~N}$-Werten der Hunde wohl darauf hinweist, dass sie etwas Fisch konsumiert haben, sind Süßwasserreservoireffekte extrem variabel, und der Effekt für den Neckar könnte viel geringer ausfallen, als hier vorgeschlagen. In Erinnerung gerufen wird auch, dass die hier vorgestellte Ernährungsanalyse auf einer eher dürftigen Vergleichsbasis der stabilen Isotope für Fisch aufbaut. Vor diesem Hintergrund wird vorgeschlagen, dass das mit IntCal13 errechnete Modell dem hier diskutierten vorzuziehen ist, da nicht genügend Informationen über den örtlichen Süßwasserreservoireffekt vorliegen. Diese Empfindlichkeitsanalyse zeigt jedoch, dass - falls eine Reservoir-Korrektur nötig sein sollte - sie die Schätzungen für den Anfang und die Laufzeit mehr beeinflusst als die Schätzungen für das Ende, und dass sie nicht größer als ein halbes Jahrhundert ausfallen würde.

\section{Diskussion}

Das oben vorgestellte bevorzugte Modell hat verschiedene Auswirkungen, sowohl auf unsere Interpretation von Klingenberg „Schlossberg“ als auch für unser Verständnis des Phänomens der MK und anderer Erdwerke. Es liefert zudem eine erste Vergleichsmöglichkeit mit anderen Methoden der Chronologiebildung, im Fall der Michelsberger Kultur durch Typologie, Seriation und Dendrochronologie.

\section{Die Geschichte von Klingenberg}

Die hier vorgestellten bayes'schen Schätzwerte für die Chronologie von Klingenberg untermauern teils frühere Interpretationen (Seidel 2008a), teils revidieren sie sie. Die sporadische Aktivität in MK II bleibt undatiert, aber die Besiedlung während MK III/IV kann jetzt zeitlich abgesteckt werden und wir konnten zeigen, dass sie wahrscheinlich über die wenigen bisher 
fassbaren Gruben auf dem Geländesporn hinausging. Eine dieser MK III/IV Gruben, 242, enthielt eine Hundedeponierung. Wurde der Platz damit schon als besonderer Ort hervorgehoben? Hundedeponierungen sind jedoch in der MK nicht auf Fundplätze mit einem Erdwerk beschränkt (Lüning 1967, 267; Seidel 2004, 152-3, Abb. 78), es ist daher fraglich inwieweit sie einen Ort als besonders kennzeichnen. In jedem Fall fanden Hundedeponierungen auf dem „Schlossberg“ nicht nur während einem Zeithorizont statt. Der „Schlossberg“ scheint auch nicht kontinuierlich genutzt worden zu sein. Das Grabensystem wurde erst für die Nutzung während MK V/Munzingen errichtet und das Modell zeigt eine Unterbrechung zwischen den Phasen MK III/IV und MK V/Munzingen an (Abb. 18; Tabelle 6: $M K I I I / I V-M K V / M z)$.

Die wesentlichen Veränderungen in der Interpretation haben sich für die Hauptnutzungsphase während MK V/Munzingen ergeben. Bisher wurde angenommen, dass ein Teil der Gruben, namentlich außerhalb des Grabensystems vor dem Bau und der Nutzung des Erdwerks angelegt wurde (Seidel 2008a, 331-2). Die modellierten Ergebnisse weisen jedoch darauf hin, dass sämtliche Gruben der Nutzungsphase MK V/Munzingen nach dem Bau des Erdwerks ausgehoben worden sein dürften und dass zumindest einige der Gruben im Innenraum des Grabenwerks noch vor den Gruben im Außenbereich genutzt wurden (Abb. 16; Tabelle 7).

Was das Erdwerk selbst betrifft, so scheint die Bauabfolge sehr rasch von statten gegangen zu sein. Die beiden Gräben dürften Teil des gleichen Bauprojektes oder aufeinanderfolgende Schritte des gleichen Vorhabens gewesen sein. Dabei wurde wahrscheinlich der äußere vor dem inneren Graben angelegt, so dass der Aushub zu einem einzigen Wall aufgeschüttet werden konnte, ohne über den Innengraben transportiert werden zu müssen. Unabhängig davon, ob es zwischen dem Bau der beiden Gräben eine Unterbrechung gab oder nicht, hätte sich der Arbeitsaufwand für das Erdwerk für einen Bautrupp von 25 Personen auf etwa drei Monate belaufen (Seidel 2008a, 226-9).

Der Bau der Grabenanlage könnte eine Reaktion auf verschiedene Bedrohungen in unruhigen Zeiten gewesen sein, die es erforderlich machten, einen gut zu verteidigenden Geländesporn mit Gräben und einem Wall abzusichern. Doch einen unumstößlichen Beweis für eine

Verteidigungsfunktion oder einen feindlichen Angriff auf die Anlage bietet selbst das Ende des Erdwerks mit den brennend in die Gräben gestürzten Holzkonstruktionen nicht. Ebenso gut könnten die Brandspuren als eine rituelle Handlung aus Anlass der Aufgabe des Erdwerkes interpretiert werden, wie sie beispielsweise aus der Geschichte der pa genannten Einhegungen der Maori auf Neuseeland bekannt sind, wenn etwa ein tapu nach dem Tod eines wichtigen Gemeinschaftsmitglieds ausgerufen wurde (Fox 1976, 11; Seidel 2008a, 384).

Die Ergebnisse des hier vorgestellten Modells werfen zudem ein neues Licht auf die Nutzung des Außenbereiches, wonach dessen Nutzung nicht als älter, sondern in einer gewissen Gleichzeitigkeit mit der Existenz des Grabensystems einzuschätzen ist. Besonderheiten, wie das Vorkommen von Tassen (Seidel 2008a, 289, Abb. 222), Miniaturäxten (Seidel 2008a, 301, Abb. 226), Spuren von Kupferverarbeitung (Seidel 2008a, 313, Abb. 248) oder der Lachsreste (Torke 2008, 456, Abb. 12), die sich alle ganz überwiegend außerhalb der Erdwerksgräben fanden, wären dann weniger chronologisch zu interpretieren. Vielmehr wären soziale Ursachen zu erwägen, etwa eine temporäre Nutzung des Außenbereichs für saisonale Ereignisse, oder gar durch eine sonst nicht in der Anlage ansässige Bevölkerung. Das Modell einer einfachen 
„Rinderaustauschstation“ dürfte wohl zu kurz greifen, da eine Dominanz des Hausrinds nicht für Fundstellen der MK allgemein festgestellt werden kann und anstelle des Austausches lebender Rinder, auch der Austausch bereits geschlachteter Tierteile zu erwägen ist (Stephan 2008, bes. 235ff.; Steppan 2003). Was den Nutzungszeitraum des Grabensystems betrifft, so ergibt sich ein Widerspruch zwischen den hier vorgeschlagenen Schätzungen von wahrscheinlich über 100 Jahren Nutzungszeit (Abb. 17; Tabelle 6: Nutzung Innengraben; Nutzung Außengraben) und dem starken Eindruck einer schnellen Verfüllung, gefolgt vom Kollaps des inneren Walles und dem Auflassen des Fundplatzes innerhalb nur weniger Jahre, wie es sich aus der Betrachtung der Befunde selbst ergibt (siehe Abb. 3). Es erscheint möglich, dass die Nutzungszeit einiger Grabenabschnitte durch Nachgrabungen bzw. Wiederausheben verlängert wurde. Allerdings gibt es dafür keine direkten Nachweise, wie gekappte Verfüllschichten. Einige Grabenabschnitte könnten während ihrer Nutzung wiederholt vollständig geräumt worden sein. Ein Argument hierfür wäre, dass die dünne Schicht aus tonigem Sediment nicht in allen Segmenten dokumentiert wurde. Da sich diese Schicht in stehendem Wasser bildete, deutet sie auf ein extrem nasses Wetterereignis unmittelbar nach dem Ausheben der betreffenden Segmente hin, während dem das Wasser nicht sofort durch den Löss hindurchsickern konnte. Dass diese Schicht in einigen Abschnitten fehlt, könnte darauf hinweisen, dass diese Segmente zeitversetzt angelegt wurden, vielleicht nur um Tage oder Wochen, oder dass sie einige Zeit nach ihrem Bau noch einmal vollständig ausgeräumt wurden, wobei ältere Schichten restlos entfernt wurden. Die zweite Möglichkeit lässt sich durch die Beobachtung untermauern, dass verstürzte Konstruktionselemente, und zwar sowohl verkohlte Hölzer als auch Steinmaterial, in genau den Abschnitten und Segmenten fehlen, die auch keine Tonschicht aufweisen. Möglicherweise wurde beides zusammen entfernt, auch wenn Konstruktionselemente manchmal auch in Abschnitten fehlen, in denen die vom Wasser abgelagerte Tonschicht dokumentiert wurde (Seidel 2008a, 225-6; Abb. 180, 181). Wurden die Abschnitte ohne diese unterste Tonschicht tatsächlich nachträglich geräumt, so müsste dies kurz nach dem Bau der Anlage passiert sein, da es beispielsweise keinen nennenswerten Unterschied zwischen der Chronologie von Grabenbereich 169 in Segment 225-169-195 im Innengraben, wo sowohl die Tonschicht als auch verstürzte Konstruktionselemente vorhanden sind, und Abschnitt 435-436-437-438-439 weiter südlich im selben Graben gibt, wo beide Schichten fehlen (Abb. 11). Abschnitt 431-432-433-433a-433b am südlichen Ende des Außengrabens ist der einzige Fall, in dem es erkennbare Hinweise auf eine Nachgrabung gegeben haben könnte. Eine Erdbrücke im Bereich 433 wurde bis zu einem gewissen Niveau unter der Oberkante des Segments gekappt und von humosen Schichten bedeckt. Das könnte bedeuten, dass der obere Teil der Erdbrücke abgegraben wurde, als das Grabenwerk selbst bereits teilweise verstürzt war und Bodenbildungsprozesse in den Gräben eingesetzt hatten (Seidel 2008a, 201). Aus keinem der Segmente dieses Abschnitts wurden Proben datiert. Die frühen Phasen der Geschichte dieser Gräben könnten also komplexer sein, als es zunächst den Anschein hat.

Auf dem „Schlossberg“ von Klingenberg fanden unterschiedliche Aktivitäten statt. Im bevorzugten Modell folgte das Ausheben von Gruben im Innenraum und deren Wiederverfüllung relativ schnell auf den Bau des Grabenwerkes. Gruben im Außenbereich wurden später angelegt (Abb. 16, 18), was auf eine ruhigere Phase des Platzes nach dem Anlegen des Erdwerkes hindeuten könnte, oder auf einen eigenen Aktivitätsrahmen, in welchem diese Gruben eine Rolle spielten. Ob eine Grube sich im Innen- oder Außenbereich befand, 
beeinflusste jedenfalls, was dort niedergelegt wurde. Einige Materialgruppen wurden ausschließlich oder fast ausschließlich im Außenbereich aufgefunden; neben den oben genannten Tassen, Tonäxten, Metallurgie- oder Lachsresten sind dies auch Gehörne von Auerochsen (Seidel 2008a, Abb. 203), und rundbodige Gefäße (Seidel 2008a, 261-2: Typen 6 und 7). Die auffällige Beschränkung etwa der Lachsreste (Torke 2008, 456, Abb. 12 ), der Kupferfunde und Schmelztiegelfragmente auf die Gruben außerhalb des Erdwerks und den äußeren Graben (Seidel 2008a, Abb. 248), könnte der Brandgefahr oder aber sozialen wie auch saisonalen Komponenten geschuldet sein. Klingenberg ist bisher der einzige bekannte Fundplatz der MK mit dem Nachweis von Kupferverarbeitung und von Fischresten, so dass Vergleiche fehlen.

Ein Eindruck zur Intensität der Nutzung der Bereiche innerhalb und außerhalb der Gräben lässt sich gewinnen, wenn die Anzahl der MK V/Mz Gruben (etwa 300; Seidel 2008a, 229) durch die Dauer der MK V/Mz Besiedlung (95-170 Jahre (95\% Wabrscheinlichkeit), wahrscheinlich 135-160 Jahre (68\% Wahrscheinlichkeit); Abb. 17: Laufzeit MK V/Mz Erdwerk und Gruben) geteilt wird. Sollten die Gruben über diesen Zeitraum in einer konstanten Zahl angelegt worden sein, dann wurde wohl nur eine, zwei oder drei Gruben pro Jahr ausgehoben. Es könnten aber auch seltener, aber dann mehrere Gruben gleichzeitig angelegt worden sein, so dass die Aktivitäten in Klingenberg ebenso gut episodisch und nicht kontinuierlich gewesen sein könnten.

Hundedeponierungen wurden die gesamte MK V/Mz Phase über angelegt (Abb. 16).

Die formalen Schätzungen unterstützen in hohem Maße eine Interpretation, wonach die Zerstörung des Erdwerkes und die Aufgabe des Außenbereiches ein einziges Ereignis darstellen. Lediglich im Innenraum wurden wahrscheinlich noch eine kurze Zeit lang weiter Gruben ausgehoben, allerdings in kleinem Maßstab. Ein einziger Endpunkt für den gesamten Fundplatz könnte für ein Szenario unruhiger Zeiten sprechen, wobei erste Bedrohungen zum Bau des Erdwerkes führten, diesem dann eine Nutzungsperiode im Innen- und Außenbereich folgte und dann ein feindlicher Angriff mit Brandereignis die endgültige Aufgabe erzwangen. Einige wenige, kleine Gedenkakte oder aber eine Nachnutzung, für die das Grabensystem nicht wesentlich war, könnten eine kurze Zeit danach stattgefunden haben. Alternativ könnte ein einziger Endpunkt — wie bereits dargelegt — auch mit einer friedlichen, geplanten Zerstörung und Aufgabe des Erdwerkes in Verbindung gebracht werden.

\section{Typologie und Chronologie Michelsberger Keramik}

Die Ergebnisse des ToTL-Projektes haben Auswirkungen auf die bisher auf die Michelsberger Keramik angewendeten Chronologiesysteme (Lüning 1967; Höhn 2002). Abbildung 19 zeigt die Schlüsselparameter für Klingenberg, zusammen mit den einzelnen Daten für Befunde mit hohen Tulpenbechern und flachbodigen Tulpenbechern. Dendrodaten, die sicher mit dem jeweiligen Keramiktyp zu verknüpfen sind, werden ebenfalls aufgeführt. Der knappe Vergleich beschränkt sich auf Jahrringdatierungen, die sowohl mit einem archäologischen Kontext als auch mit charakteristischen Keramikfunden der MK korreliert werden können. Die Datierungen für Fundplätze wie Thayngen „Weiher“ und Nußbaumer See „Inseli“ werden daher ausgeschlossen.

Die Jahrringdatierungen der Bauphase IB von Ehrenstein in Oberschwaben mit einem Fälldatum von 3955 BC (Billamboz 1992, 87) ergeben ein Datum für das Ende von MK II, das kompatibel 
mit dem hier angenommenen Beginn der MK III/IV Gruben in Klingenberg ist (Beginn MK III/IV Gruben; Abb. 19).

Die geschätzte Dauer der MK III/IV Besiedlung in Klingenberg stimmt gut mit den dendrochronologisch erfassten Zeitspannen für charakteristische Formen der Stufen MK III und MK IV überein, die jüngst durch Matuschik (2011, 271-4) herausgearbeitet wurden. Für Seeufersiedlungen in Südwestdeutschland liegen demnach Dendrodaten zwischen 3919 und 3834 BC für Inventare mit Ösenkranzflaschen mit tief sitzenden Ösen vor, die MK III charakterisieren, während für Kontexte mit Ösenleistenflaschen, die für MK IV definierend sind, Dendrodaten zwischen 3869 und 3817 BC verfügbar sind. Demnach verbleibt für die Dauer der Stufe MK III nur etwa ein Jahrhundert, anstelle der bislang angenommen drei Jahrhunderte (Höhn 2002, 193). Zudem überlappen sich die Laufzeiten der beiden Flaschentypen; und nach wie vor besteht ein Problem, MK III und MK IV typologisch nur aufgrund der An- bzw. Abwesenheit der seltenen Ösenleistenflaschen zu trennen. Aus diesem Grund wurde die Keramik beider Stufen hier zu MK III/IV zusammengefasst (wie auch bei Seidel 2008a, 331, 388, Anhang 2; dies entspricht Höhns (2002) „Intervall 3c").

Einige Details zur absoluten Datierung und zum Zeitraum der MK V/Munzingen Aktivitäten in Klingenberg verdienen es, hervorgehoben zu werden. Gruben, die Keramik mit typologischen Elementen der Stufen MK IV und MK V enthielten, führten zur Interpretation, dass diese Gruben vor dem Bau des Erdwerks ausgehoben worden sein könnten (Seidel 2008a, 287-91). Die im Rahmen des ToTL-Projekts durchgeführten Datierungen für drei dieser Inventare, alle an Hunde(teil)skeletten und alle von außerhalb des Erdwerks (Abb. 13: SUERC-52391, -52395, TS 46), erlauben es, den Beginn und das Ende der MK IV/V Inventare abzuschätzen (Abb. 19: Beginn MK IV/V Gruben außerbalb des Erdwerks, Ende MK IV/V Gruben außerbalb des Erdwerks). Interessanterweise fallen deren absolute Daten in, und nicht vor; die Zeitspanne für MK V/Munzingen Inventare ohne MK IV Elemente (Abb. 19: Beginn MK V/Mz Gruben außerhalb des Erdwerks, Ende $M K V / M_{z}$ Gruben außerhalb des Erdwerks). Damit dürften die MK IV/V Inventare zeitgleich mit den MK V/Munzingen Inventaren sein.

Höhn $(2002,181)$ nahm für Klingenberg mehrere Besiedlungsphasen an, da sich acht Grubeninventare über ihre Seriationsintervalle $4 \mathrm{~b}$ und $4 \mathrm{c}$ verteilten, die das späteste Michelsberg abdecken. Diese Trennung der Intervalle 4b und 4c konnte bei Seriationen des Gesamtinventars von Klingenberg nicht bestätigt werden. Grundsätzlich konnte das Gesamtinventar von Klingenberg nicht in stilistische Phasen oder gar Besiedlungsphasen unterteilt werden (Seidel 2008a, 295-8 und 317). Die vorgelegten absoluten Daten unterstützen somit die auf Basis der Seriationen aufgestellte Hypothese einer Gleichzeitigkeit von MK IV und MK V/Munzingen Elementen.

Obwohl Tulpenbecher in allen Bereichen des Fundplatzes zahlreich auftreten (Seidel 2008a, Abb. 221), sind nur fünf Datierungen direkt mit ihnen assoziiert. Diese Datierungen weisen darauf hin, dass Tulpenbecher bis zum Ende der Besiedlung weiter genutzt wurden, parallel neben den flachbodigen Gefäßen, die als Munzinger Formen angesehen werden (Abb. 19).

Eine mutmaßliche Phase „MK VI“ ohne Tulpenbecher, wie durch Biel (1987) vorgeschlagen, kann auf Grundlage der ${ }^{14} \mathrm{C}$-Chronologie von Klingenberg ausgeschlossen werden, wie dies 
bereits auf der Basis der Seriationen von Höhn $(2002,181)$ und Seidel $(2008 a, 298)$ erfolgte. Beim Fehlen von Tulpenbechern im spätesten MK Intervall („Intervall 4c“, Höhn 2002, 183) im Bodenseegebiet und im Oberrheintal dürfte es sich demnach um ein regionales Spezifikum handeln.

Lüning (1967, 91-7; vgl. Jeunesse 1989) sah Munzingen als eine oberrheinische Regionalgruppe an, die er zeitgleich mit MK III und MK IV ansetzte; und für die er keinen Hinweis für ein Andauern noch während MK V hatte. Höhn (2002, 171-81, Abb. 169) konnte mit neuerem Material dann zeigen, dass flachbodige Keramik charakteristisch für das Ende der MK Keramikentwicklung ist, d.h. für MK V und Munzingen, und dass erste Flachböden bereits mit Elementen auftreten, die die Stufe MK IV charakterisieren. Diese Einschätzung wurde mit dem Klingenberger Material bestätigt (Seidel 2008a, 249-99), für das keine Trennung von Gruben mit MK und Munzingen Inventaren vorgenommen werden konnte. Munzingen ist vielmehr als „Stil“ aufzufassen, der zum klassischen Formenspektrum der MK hinzutritt. Dies scheint auch im Rheintal der Fall zu sein. Im unteren Elsass und der Kaiserstuhlregion Baden-Württembergs kann der Beginn der Munzinger Keramik typologisch auf die Stufe MK III/IV bzw. den Anfang von MK IV fixiert werden (Dieckmann 1991, 207; Höhn 2002, 233; Lefranc 2001, 121-4), wohingegen aus dem Neckarland noch kein Inventar bekannt ist, das hier den Beginn von Munzingen repräsentieren könnte (Seidel 2008a, 321-3).

Ösenleistenflaschen, die die Stufe MK IV definieren, wurden bisher an keinem Fundplatz gefunden, der später als 3817 BC datiert (Matuschik 2011, 280). Im Formenbestand der MK werden sie durch eine Reihe flachbodiger Flaschenvarianten mit kurzem Hals, meist ohne Ösen abgelöst (Höhn 2002, Abb. 198: „F4“). Diese Flaschen hat Michelsberg mit Pfyn und PfynAltheim gemeinsam und sie sind auch im Klingenberger Material zu finden (Seidel 2008b, Taf. 107.6; 117.6; 136.2; 152.5; 155.1; 162.3,5). In den Fundplätzen des Alpenvorlandes treten die flachbodigen Flaschen („,F4“) ab etwa 3800 BC auf (Zürich „Seefeld“ Schicht 9: 3804-3827 BC = frühes Pfyn) (Kolb 2003, 42), was gut zur Datierung der MK V/Munzingen Belegung von Klingenberg passt.

Für Keramik der Pfyn-Altheimer Gruppe, die Ähnlichkeiten mit der MK V/Munzingen Keramik | von Klingenberg aufweist, -liegen von Fundplätzen im südwestdeutschen Federseegebiet dendrochronologische Datierungen der Kategorie A vor, die zwischen den Jahren 3745 und 3688 BC liegen (Billamboz 1998, 164) (Abb. 19). Eine Munzinger Gefäßform (Topf Typ 12,2) von Sipplingen am Bodensee aus Schicht 9, vergesellschaftet mit flachbodigen Flaschen, wird von einem liegenden Pfosten auf 3689 BC datiert (Matuschik 2011, 279, Abb. 183; Kolb 2003, 33-7, Taf. 6,20 und Taf. 7, 29-30; vgl. Seidel 2008a, 326-30).

Damit wird klar, dass in Zukunft eine Kombination verschiedener Herangehensweisen notwendig ist, insbesondere für Gebiete, in denen keine präzise Datierung durch die Dendrochronologie möglich ist. Die typologisch ermittelten Ordnungssysteme müssen nicht immer chronologisch sein. Diese Methoden und die quantifizierten, probabilistischen Schätzwerte für Datierungen, wie sie mittels formaler Modelle aus Radiokarbondaten an stringent ausgewählten Materialien errechnet werden, können effektiv miteinander kombiniert werden. 
Weitreichendere Auswirkungen

Die Datierung von Klingenberg ist auf drei über den Fundplatz hinausgreifenden Ebenen relevant: Für die Region, in der sich der Fundplatz befindet; allgemein für den Charakter und die Verbreitung der Michelsberger Erdwerke und das Phänomen der Ostdrift der MK (vgl. Geschwinde 2013); und weiter entfernt für das Auftreten segmentierter Grabenwerke in Südengland ab den letzten Jahrzehnten des 38. Jahrhunderts cal BC (Whittle et al. 2011).

In einigen Regionen der MK finden sich lokal Konzentrationen von Erdwerken, beispielsweise im Neckarland und im Kraichgau im nördlichen Baden-Württemberg; am nördlichen Oberrhein und weiter abwärts in der Kölner Bucht; um Soest in Westfalen; in Ostfrankreich in den Tälern der Aisne und der Oise; oder im Braunschweiger Harzvorland in Niedersachsen (Abb. 1). In diesen Gebieten finden sich drei, vier oder mehr Erdwerke in Abständen von weniger als $1 \mathrm{~km}$ bis $15 \mathrm{~km}$ voneinander entfernt (Meyer und Raetzel-Fabian 2006). Das Neckartal um Heilbronn, wo sich Klingenberg befindet, ist ein eindrucksvolles Beispiel hierfür (Abb. 20). Wo solche Erdwerkskonzentrationen datiert wurden, wenn auch nur über die assoziierten Funde, scheinen nicht alle Anlagen gleichzeitig genutzt worden zu sein.

So weist das Material von Klingenberg „Schlossberg“, „Hetzenberg“ und „Ilsfeld“ im Neckartal (Seidel 2008a; Schlenker et al. 2008) und von „Michelsberg“, „Aue“, „Scheelkopf“ und „Altenberg“ bei Bruchsal im Kraichgau darauf hin, dass die Erdwerke in diesen "Erdwerkszentren" nicht alle zur selben Zeit in Nutzung waren (Seidel 2008a; Regner-Kamlah und Seidel in Vorber.). In die Stufe MK V/Munzingen können in Baden-Württemberg fünf Erdwerke datiert werden: im Neckarland Klingenberg selbst und Leonberg „Silberberg“ nahe Stuttgart, bei Bruchsal der „Scheelkopf“ und möglicherweise der namengebende „Michelsberg“, weiter südlich im Oberrheintal bei Freiburg das eponyme Erdwerk von Munzingen „Tuniberg“ (Abb. 20; Tabelle 8; Reiter 2005; Steppan 2003; Seidel 2008a; Kimmig 1947; Maier 1958; Schmid 1958). Offenbar wurden alle Erdwerke der Phase MK V/Munzingen neu auf zuvor nicht umhegten Plätzen angelegt (Seidel 2008a, 341-7). Eine gewisse Sonderstellung nimmt dabei der namengebende „Michelsberg“ ein, auf dem ein Erdwerk sicher ab MK IV (MK III/IV) nachzuweisen ist, es jedoch ungeklärt bleibt, ob die Anlage während MK V/Munzingen weiter bestand. Diese Neugründung von Erdwerken könnte ein weiteres Indiz für Veränderungen am Ende der MK sein, und es könnte ein funktionaler Unterschied zwischen den größeren Erdwerken der frühen MK und den kleinen Anlagen der späten MK bestehen (Seidel 2008a, 389). Die ausgedehnten Erdwerke der „klassischen“ MK (d.h. MK II bis MK III/IV) wurden mitunter wiederholt aufgesucht, beispielsweise im Fall von Ilsfeld oder Bruchsal „Aue“. Andere Erdwerksplätze wurden nach kurzer Zeit aufgegeben, wie Neckarsulm-Obereisesheim „Hetzenberg“ oder Bruchsal „Altenberg“ (beide Anlagen ergaben ausschließlich Anhaltspunkte für MK II; Lüning 1967, 233-7; Koch 2005; Seidel 2008a, 11-70). Für Baden-Württemberg wurde daher ein „Heilbronner Modell“ (Seidel 2008a, 388; Jacob 2010, 51) bzw. „HeilbronnKraichgau“-Modell vorgeschlagen (Regner-Kamlah und Seidel in Vorb.), wonach die Nutzungszeit eines Erdwerks eher kurz war und die Aktivitäten zwischen den Erdwerken einer Kleinregion wechselten. Die Erdwerke werden in diesem Rahmen als wichtige Treffpunkte für eher kleine und relativ wenig ortsfeste Sozialverbände angesehen. Eine chronologische 
Zusammenschau lässt einen spezifischen Horizont am Ende der MK erkennen, in dem sie eine Veränderungsphase durchläuft, die mit Kontakten zum Néolithique Moyen Bourguignon (NMB), den Gruppen Pfyn, Pfyn-Altheim und Altheim im Süden, Südwesten und Südosten und zu Baalberge und den Trichterbechergruppen im Norden verbunden ist.

Zum Konzept einer Nordostbewegung der MK passt die Neuentdeckung zahlreicher Erdwerke an der nordöstlichen Peripherie des Gebiets der MK (Geschwinde und Raetzel-Fabian 2009; Geschwinde 2013, 197). Hier überlappt sich das Verbreitungsgebiet der Erdwerke der MK mit dem der Erdwerke der TBK (Andersen 1990; Müller 2010). Diese komplexe Sachlage wirft noch viele Fragen zur Ansprache und Datierung einzelner Fundplätze in dieser Region auf, die wir hier nicht im Detail behandeln können. Für den Augenblick gilt es aber festzuhalten, dass das Konzept einer fortschreitenden Nordostdrift der MK im Grundsatz zutreffend erscheint, und es von Interesse ist, die Ausbreitung der MK in ihren Auswirkungen auf und in ihren Beziehungen zu den „Kernregionen“, d.h. Regionen mit alter MK-Präsenz zu untersuchen, da sich hier Regionen übergreifende Veränderungen am Ende der Michelsberger Kultur im 38. und 37. Jahrhundert cal BC abzeichnen.

Obwohl dies noch weiter weg führt, erscheint es schlussendlich doch nachdenkenswert, welche Beziehungen zwischen dem Bau eines Erdwerkes wie Klingenberg in einem entfernten Seitental des Rheins am Übergang vom 39. zum 38. Jahrhundert cal BC, und dem Auftreten segmentierter Grabenanlagen in Südengland wahrscheinlich im späten 38. Jahrhundert cal BC bestehen. Obwohl die südenglischen Beispiele nicht identisch sind mit ihren kontinentalen Vorläufern, haben sie doch so viele Eigenschaften mit diesen gemeinsam, dass erstere in irgendeiner Weise, und wenn nur als generelle Idee, wie man etwas zu tun hat, von letzteren abgeleitet sein müssen (Whittle et al. 2011, 878-85). Ein Desiderat bleiben zu dieser Frage nach wie vor präzisere Datierungen für nordfranzösische Fundplätze, wobei es dort Probleme mit der Kollagenerhaltung in Knochenfunden aus Flussschottern gibt (Jérôme Dubouloz, pers. Mitt.). Die späte Datierung von Klingenberg bringt die südenglischen Grabenwerke jedenfalls in eine Zeitspanne, innerhalb derer eine aktive soziale Erinnerung zwischen diesen entfernten Gemeinschaften möglich wird. Es bleibt jedoch ein Rätsel, warum dieses Phänomen, das als „Westdrift“ bezeichnet werden könnte, erst so spät und nicht früher im 38. Jahrhundert cal BC beginnt. Mittlerweile besteht ein ganzer Katalog dringender Fragen zum 38. Jahrhundert cal BC, der die Gemeinschaften von Südengland bis in die östlichen Verbreitungsgebiete der MK einschließt. Dass wir inzwischen aber in der Lage sind, diesen spezifischen Horizont überhaupt zu fassen, ist ein signifikanter Fortschritt für unser Verständnis dieser Zeit.

Nicht nur die südenglischen Grabenwerke sind selbstverständlich für die Gesamtgeschichte der Erbauung und Nutzung von Erdwerken im späten fünften und frühen vierten Jahrtausend cal $\mathrm{BC}$ in ganz Westeuropa von Belang. Es gibt im gesamten umrissenen Gebiet Herausforderungen, von der Präzisierung der Ereignisabfolgen in einzelnen Erdwerken, wie denjenigen der Michelsberger Kultur, bis hin zur Erarbeitung einer grundlegenden Chronologie der Erdwerke in davon weit entfernten Gebieten, etwa in Südskandinavien (Klassen 2014; Andersen 2015). Die Details, die in diesem Beitrag für ein relativ bescheiden dimensioniertes Erdwerk der Michelsberger Kultur vorgestellt wurden, mögen sehr ausführlich erscheinen, aber sie sind unumgänglich. Diese eine Studie hat dazu beigetragen, eine robustere, argumentativ 
belastbarere und umfassendere Geschichte eines Fundplatzes aufzubauen. Sie hat aber auch gezeigt, wie viel Arbeit für die zukünftige Forschung noch ansteht.

\section{Danksagungen}

Unser Dank gilt den folgenden Personen: Patrizia Schlemper, Zentrales Fundarchiv des Archäologischen Landesmuseum Baden-Württemberg in Rastatt für die Bereitstellung von Funden; Seren Griffiths für ihre Hilfe bei der Auswahl und Entnahme der Proben; John Meadows für die Übermittlung weiterer Einzelheiten zu den in Kiel datierten Proben; Daniela Hofmann für die Übersetzung des Textes ins Deutsche; Anthony Denaire für die Übersetzung des Resumées ins Französische. Die Beprobung wäre nicht möglich gewesen ohne die kuratorische Betreuung der Archive in Rastatt (Osteologisches Archiv des Landesamts für Denkmalpflege) und im Institut für Botanik der Universität Hohenheim. The Times of Their Lives (www.totl.eu) wird mit einem Advanced Investigator Grant (295412) des European Research Council unterstützt und von Alasdair Whittle (Cardiff University) und Alex Bayliss (Historic England) geleitet.

\section{Literatur}

Andersen 1990: N. Andersen, Sarup volume 1. The sarup enclosures (Moesgaard 1990).

Andersen 2015: N. H. Andersen, Causewayed enclosures in northern and western Europe. In: C. Fowler/J. Harding/D. Hofmann (Hrsg.), The Oxford handbook of Neolithic Europe (Oxford 2015) 795-812.

Ashmore 1999: P. Ashmore, Radiocarbon dating: avoiding errors by avoiding mixed samples. Antiquity 73, 1999, 124-30.

Bayes 1763: T. R. Bayes, An essay towards solving a problem in the doctrine of chances. Philosophical Transactions of the Royal Society 53, 1763, 370-418.

Bayliss 2009: A. Bayliss, Rolling out revolution: using radiocarbon dating in archaeology. Radiocarbon 51, 2009, 123-47.

Bayliss et al. 2007: A. Bayliss/C. Bronk Ramsey/J. van der Plicht/A. Whittle, Bradshaw and Bayes: towards a timetable for the Neolithic. Cambridge Archaeological Journal 17.1, supplement, 2007, 1-28. 
Bayliss et al. 2004: A. Bayliss/E. Shepherd Popescu/N. Beavan-Athfield/C. Bronk Ramsey/G. T. Cook/A. Locker, The potential significance of dietary offsets for the interpretation of radiocarbon dates: an archaeologically significant example from medieval Norwich. Journal of Archaeological Science 431, 2004, 563-75.

Bayliss et al. 2011: A. Bayliss/J. van der Plicht/C. Bronk Ramsey/G. McCormac/F. Healy/A. Whittle, Towards generational time-scales: the quantitative interpretation of archaeological chronologies. In: A. Whittle/F. Healy/A. Bayliss, Gathering time: dating the Early Neolithic enclosures of southern Britain and Ireland (Oxford 2011) 16-60.

Beavan Athfield et al. 2008: N. Beavan Athfield/R. C. Green/J. Craig/B. McFadgen/S. Bickler, Influence of marine sources on ${ }^{14} \mathrm{C}$ ages: isotopic data from Watom Island, Papua New Guinea inhumations and pig teeth in light of new dietary standards. Journal of the Royal Society of New Zealand 38, 2008, 1-23.

Biel 1987: J. Biel, Abschließende Untersuchung eines Michelsberger Erdwerks bei HeilbronnKlingenberg. Archäologische Ausgrabungen in Baden-Württemberg 1987, 50-4.

Biel et al. 1998: J. Biel/H. Schlichtherle/M. Strobel/A. Zeeb (Hrsg.), Die Michelsberger Kultur und ihre Randgebiete - Probleme der Entstehung, Chronologie und des Siedlungswesens. Kolloquium Hemmenhofen 21-23.2.1997. Jens Lüning zum 60. Geburtstag. Materialhefte zur Archäologie in Baden-Württemberg 43 (Stuttgart 1998).

Billamboz 1992: A. Billamboz, Erste dendrochronologische Zeitmarken für die jungneolithische Talsiedlung Ehrenstein, Alb-Donau-Kreis. Archäologische Ausgrabungen in BadenWürttemberg 1992, 84-7.

Billamboz 1998: A. Billamboz, Die jungneolithischen Dendrodaten der Pfahlbausiedlungen Südwestdeutschlands als Zeitrahmen für die Einflüsse der Michelsberger Kultur in ihren südlichen Randgebiet. In: J. Biel et al. (Hrsg.) 1998, 159-68.

Bonsall et al. 2015: C. Bonsall/R. Vasić/A. Boroneanţ/M. Roksandić/A. Soficaru/K. McSweeney/A. Evatt/Ü. Aguraiuja/C. Pickard/V. Dimitrijević/T. Higham/D. Hamilton/G. 
Cook, New AMS ${ }^{14} \mathrm{C}$ dates for human remains from stone age sites in the Iron Gates reach of the Danube, south-east Europe. Radiocarbon 57, 2015, 33-46.

Brock et al. 2010: F. Brock/T. Higham/P. Ditchfield/C. Bronk Ramsey, Current pretreatment methods for AMS radiocarbon dating at the Oxford Radiocarbon Accelerator Unit (ORAU). Radiocarbon 52, 2010, 103-12.

Bronk Ramsey 1995: C. Bronk Ramsey, Radiocarbon calibration and analysis of stratigraphy. Radiocarbon 36, 1995, 425-30.

Bronk Ramsey 2001: C. Bronk Ramsey, Development of the radiocarbon calibration program. Radiocarbon 43, 2001, 355-63.

Bronk Ramsey 2009: C. Bronk Ramsey, Bayesian analysis of radiocarbon dates. Radiocarbon 51, 2009, 337-60.

Bronk Ramsey/Lee 2013: C. Bronk Ramsey/S. Lee, Recent and planned developments of the program OxCal. Radiocarbon 55, 2013, 720-30.

Brown et al. 1988: T. A. Brown/D. E. Nelson/J. S. Vogel/J. R. Southon, Improved collagen extraction by modified Longin method. Radiocarbon 30, 1988, 171-7.

Buck et al. 1996: C. E. Buck/W. G. Cavanagh/C. D. Litton, Bayesian approach to interpreting archaeological data (Chichester 1996).

Buck et al. 1992: C. E. Buck/C. D. Litton/A. F. M. Smith, Calibration of radiocarbon results pertaining to related archaeological events. Journal of Archaeological Science 19, 1992, 497-512.

Dieckmann 1991: B. Dieckmann, Zum Mittel- und Jungneolithikum im Kaiserstuhlgebiet. University of Freiburg/Breisgau: unpublished dissertation 1991.

Dörr et al. 1989: H. Dörr/B. Kromer/K. O. Münnich, Fast ${ }^{14} \mathrm{C}$ sample preparation of organic material. Radiocarbon 31, 1989, 264-8. 
Durrwachter et al. 2006: C. Durrwachter/O. E. Craig/M. J. Collins/J. Burger/K. W. Alt, Beyond the grave: variability in Neolithic diets in southern Germany? Journal of Archaeological Science 33, 2006, 39-48.

Ewersen/Ziegler 2014: V. J. Ziegler/S. Ziegler, Hungrige Hunde jagen am besten: Nahrungsgrundlagen mesolithischer und neolithischer Hunde nach Isotopenverhältnissen. Offa 67/68, 2014, 5-26

Fernandes 2014: R. Fernandes/A. R. Millard/M. Brabec/M.-J. Nadeau/P. Grootes, Food reconstruction using isotopic transferred signals (FRUITS): a Bayesian model for diet reconstruction. 2014. PLoS ONE 9(2): e87436. doi:10.1371/journal.pone.0087436

Fox 1976: A. Fox, Prehistoric Maori fortifications in the North Island of New Zealand (Auckland 1976).

Freeman et al. 2010: S. P. H. T. Freeman/G. T. Cook/A. B. Dougans/P. Naysmith/K. M. Wicken/S. Xu, Improved SSAMS performance. Nuclear Instruments and Methods in Physics Research B 268, 2010, 715-17.

Geschwinde 2013: M. Geschwinde, Erdwerke des 5. und 4. Jahrtausends v. Chr. In: H. Meller (Hrsg.), 3300 BC: mysteriöse Steinzeittote und ihre Welt, 195-201 (Mainz 2013).

Geschwinde/Raetzel-Fabian 2009: M. Geschwinde/D. Raetzel-Fabian, EWSBSL - Eine Fallstudie zu den jungneolithischen Erdwerken am Nordrand der Mittelgebirge (Rahden/Westf. 2010).

Grootes et al. 2004: P. M. Grootes/M.-J. Nadeau /A. Rieck, ${ }^{14}$ C-AMS at the Leibniz-Labor: Radiometric dating and isotope research. Nuclear Instruments and Methods in Physics Research Section B 223-224, 2004, 55-61.

Höhn 2002: B. Höhn, Die Michelsberger Kultur in der Wetterau (Bonn 2002).

Jacob 2010: C. Jacob, Steinzeit - Grossbaustellen // Befestigte Siedlungen im Heilbronner Land. Städtische Museen Heilbronn. Museo 25, 2010 (Heilbronn 2010). 
Jeunesse 1989 : C. Jeunesse, La culture de Munzingen dans le cadre du «Jungneolithikum» du sud-ouest de l'Europe centrale d'après les découvertes récentes des sites alsacaciens de Didenheim (Ht-Rhin) et Geispolsheim (B-Rhin). Cahiers de l'Association pour la Promotion de la Recherche Archéologique en Alsace 5, 1989, 155-84.

Jeunesse/Seidel 2010: C. Jeunesse/U. Seidel, Die Erdwerke. In Badisches Landesmuseum Karlsruhe (Hrsg.), Jungsteinzeit im Umbruch. Die „Michelsberger Kultur“ und Mitteleuropa vor 6000 Jahren. (Darmstadt 2010) 58-61.

Jones/Nicholls 2001: M. Jones/G. Nicholls, Reservoir offset models for calibration. Radiocarbon 43, 2001, 119-24.

Keaveney/Reimer 2012: E. M. Keaveney/P. J. Reimer, Understanding the variability in freshwater radiocarbon offsets. Journal of Archaeological Science 39, 2012, 1306-16.

Kimmig 1947: W. Kimmig, Neue Michelsbergfunde am Oberrhein. Badische Fundberichte 17, 1947, 95-127.

Klassen 2014: L. Klassen, Along the road: aspects of causewayed enclosures in south Scandinavia and beyond (Aarhus 2014).

Koch2005: R. Koch, Das Erdwerk der Michelsberger Kultur auf dem Hetzenberg bei Heilbronn-Neckargartach. Forschungen und Berichte zur Vor- und Frühgeschichte in BadenWürttemberg 3. Teil I: Befunde und Funde (Stuttgart 2005).

Kolb 2003: M. Kolb, Siedlungen der Pfyner Kultur im Osten der Pfahlbaubucht von Sipplingen, Bodenseekreis. Band 1: Funde und Befunde (Gaienhofen-Hemmenhofen/Freiburg 2003).

Kromer et al. 1992: B. Kromer/K.-O. Münnich, $\mathrm{CO}_{2}$ gas proportional counting in radiocarbon dating - review and perspective. Radiocarbon after Four Decades. R. E. Taylor, A. Long and R. S. Kra (New York 1992) 184-197.

Kromer et al. 2013: B. Kromer/S. Lindauer/H.-A. Synal/L. Wacker, MAMS - A new AMS

facility at the Curt-Engelhorn-Centre for Archaeometry, Mannheim, Germany. Nuclear 
Instruments and Methods in Physics Research Section B: Beam Interactions with Materials and Atoms 294, 2013, 11-13.

Lanting/van der Plicht 1998: J. N. Lanting/J. van der Plicht, Reservoir effects and apparent ages. Journal of Irish Archaeology 9, 1998, 151-65.

Lefranc 2001: P. Lefranc, L'habitat néolithique moyen et récent de Holzheim 'Altmatt'/Zone d'Activités Économiques Phase 3 (Bas-Rhin), fouilles 2000 et 2011. Cahiers de l'Association pour la Promotion de la Recherche Archéogique en Alsace 17, 2001, 107-34.

Lindley 1985: D. V. Lindley, Making decisions (second edition) (London 1985).

Lüning 1967: J. Lüning, Die Michelsberger Kultur. Ihre Funde in zeitlicher und räumlicher Gliederung. Bericht der Römisch-Germanischen Kommission 48, 1967, 1-350.

Maier 1958: R. A. Maier, Neufunde aus der „Michelsberger“ Höhensiedlung bei Munzingen, Lkr. Freiburg im Breisgau. Badische Fundberichte 21, 1958, 7-40.

Matuschik 1991: I. Matuschik, Grabenwerke des Spätneolithikums in Süddeutschland. Fundberichte aus Baden-Württemberg 16, 1991, 27-55.

Matuschik 2011: I. Matuschik, Die Keramikfunde von Hornstaad-Hörnle I-VI.

Besiedlungsgeschichte der Fundstelle und Keramikentwicklung im beginnenden 4. Jahrtausend im Bodenseeraum. Siedlungsarchäologie im Alpenvorland XII. Forschungen und Berichte zur Vor- und Frühgeschichte in Baden-Württemberg 122 (Stuttgart 2011).

Meyer/Raetzel-Fabian 2006: M. Meyer/D. Raetzel-Fabian, Neolithische Grabenwerke in Mitteleuropa: Ein Überblick. www.jungsteinSITE.de, 15. Dezember 2006, 1-54.

Mook/Waterbolk 1985: W. G. Mook/H. T. Waterbolk, Radiocarbon dating (Strasbourg 1985).

Müller 2010: J. Müller, Dorfanlagen und Siedlungssysteme. Die europäische Perspektive: Südosteuropa und Mitteleuropa. In: Badisches Landesmuseum (Hrsg.), Jungsteinzeit im Umbruch: die »Michelsberger Kultur« und Mitteleuropa vor 6.000 Jahren (Karlsruhe 2010) 250_ 7. 
Münnich 1957: K. O. Münnich, Heidelberg Natural Radiocarbon Measurements I. Science 126, 1957, 194-9.

Nadeau et al. 1997: M.-J. Nadeau/M. Schleicher/P. M. Grootes/H. Erlenkeuser/A.

Gottdang/D.J.W. Mous/J. M. Sarnthein/H. Willkomm, The Leibniz-Labor facility at the Christian-Albrechts University, Kiel, Germany. Nuclear Instruments and Methods in Physics

Research B 123, 1997, 22-30.

Nadeau et al. 1998: M.-J. Nadeau/P. M. Grootes/M. Schleicher/P. Hasselberg/A. Rieck/M. Bitterling, Sample throughput and data quality at the Leibniz-Labor AMS facility. Radiocarbon 40, 1998, 239-45.

Petrasch 2015: J. Petrasch, Central European enclosures. In: C. Fowler/J. Harding/D. Hofmann (Hrsg.), The Oxford handbook of Neolithic Europe (Oxford 2015) 763-78.

Regner-Kamlah/Seidel in press: B. Regner-Kamlah/U. Seidel, The Michelsberg culture of northern Baden-Württemberg — a case study of a landscape with enclosures and open sites. Meeting Megaliths- Societies-Landscapes. Early Monumentality and social differentiation in Neolithic Europe. Kiel 16.-20.06.2015 (in press).

Reimer et al. 2013: P. J. Reimer/E. Bard/A. Bayliss/ J. W. Beck/P. G. Blackwell/C. Bronk Ramsey/C. E. Buck/H. Cheng/R. L. Edwards/M. Friedrich/P. M. Grootes/T. P. Guilderso/H. Haflidason/I. Hajdas/C. Hatté/T. J. Heaton/D. L. Hoffmann/A. G. Hogg/K. A. Hughen/K. F. Kaiser/B. Kromer/S. W. Manning/M. Niu/R. W. Reimer/D. A. Richards/E. M. Scott/J. R. Southon/R. A. Staff/C. S. M. Turney/J. van der Plicht, Intcal 13 and marine13 radiocarbon age calibration curves 0-50,000 years cal BP. Radiocarbon 55, 2013, 1869-87.

Reiter 2005: S. Reiter, Die beiden Michelsberger Anlagen von Bruchsal ‘Aue’ und Bruchsal 'Scheelkopf'. Zwei ungleiche Nachbarn. Materialhefte zur Archäologie in Baden-Württemberg 65 (Stuttgart 2005). 
Roth/Hobson 2000: J. D. Roth/K. A. Hobson, Stable carbon and nitrogen isotopic fractionation between diet and tissue of captive red fox: implications for dietary reconstruction. Canadian Journal of Zoology 78, 2000, 848-52.

Sayle et al. 2014: K. L. Sayle/G. T. Cook/P. L. Ascough/H. Gestsdóttir/W. D. Hamilton /T. H. McGovern, Utilization of $\delta^{13} \mathrm{C}, \delta^{15} \mathrm{~N}$, and $\delta^{34} \mathrm{~S}$ analyses to understand ${ }^{14} \mathrm{C}$ dating anomalies within a Late Viking Age community in Northeast Iceland. Radiocarbon 56, 2014, 811-21.

Schlenker et al. 2008: B. Schlenker/E. Stephan/J. Wahl, Michelsberger Erdwerke im Raum Heilbronn. Neckarsulm-Obereisesheim 'Hetzenberg' und Ilsfeld 'Ebene', Lkr. Heilbronn, Heilbronn-Klingenberg 'Schlossberg', Stadtkreis Heilbronn. Band 3: Osteologische Beiträge (Stuttgart 2008).

Schmid 1958: E. Schmid, Die „Nebenfunde“ auf dem Munzinger Berg. Badische Fundberichte 21, 1958, 41-76.

Schoch et al. 1980: H. Schoch/M. Bruns/K. O. Münnich/M. Münnich, A multi-counter system for high precision carbon-14 measurements. Radiocarbon 22, 1980, 442-7.

Scott 2003: E. M. Scott, The third international radiocarbon intercomparison (TIRI) and the fourth international radiocarbon intercomparison (FIRI) 1990-2002: results, analyses, and conclusions. Radiocarbon 45, 2003, 135-50.

Scott et al. 2007: E. M. Scott/G. T. Cook/P. Naysmith/C. Bryant/D. O’Donnell, A report on phase 1 of the 5 th international radiocarbon intercomparison (VIRI). Radiocarbon 49, 2007, 409-26.

Scott et al. 2010a: E. M. Scott/G. T. Cook/P. Naysmith, A report on phase 2 of the fifth international radiocarbon intercomparison (VIRI). Radiocarbon 52, 2010, 846-58.

Scott et al. 2010b: E. M. Scott/G. T. Cook/P. Naysmith, The Fifth Radiocarbon Intercomparison (VIRI): an assesssment of laboratory performance in stage 3. Radiocarbon 52, 2010, 859-65. 
Seidel 2004: U. Seidel, Die jungneolithischen Siedlungen von Leonberg-Höfingen, Kr.

Böblingen. Materialhefte zur Archäologie in Baden-Württemberg 69 (Stuttgart 2004).

Seidel 2008a: U. Seidel, Michelsberger Erdwerke im Raum Heilbronn. Neckarsulm-

Obereisesheim 'Hetzenberg' und Ilsfeld 'Ebene', Lkr. Heilbronn, Heilbronn-Klingenberg

'Schlossberg', Stadtkreis Heilbronn. Materialhefte zur Archäologie in Baden-Württemberg 81/1:

Text, Literatur und Anhänge (Stuttgart 2008).

Seidel 2008b: U. Seidel, Michelsberger Erdwerke im Raum Heilbronn. Neckarsulm-

Obereisesheim 'Hetzenberg' und Ilsfeld 'Ebene', Lkr. Heilbronn, Heilbronn-Klingenberg

'Schlossberg', Stadkreis Heilbronn. Materialhefte zur Archäologie in Baden-Württemberg 81/2:

Kataloge und Tafeln (Stuttgart 2008).

Slota et al. 1987: P. J. Slota/A. J. T. Jull/T. W. Linick/L. J. Toolin, Preparation of small samples for ${ }^{14} \mathrm{C}$ accelerator targets by catalytic reduction of CO. Radiocarbon 29, 1987, 303-6.

Steier/Rom 2000: P. Steier/W. Rom, The use of Bayesian statistics for 14C dates of chronologically ordered samples: a critical analysis. Radiocarbon 42, 2000, 183-98.

Stephan 2008: E. Stephan, Die Tierknochenfunde aus Heilbronn-Klingenberg 'Schlossberg’. In: B. Schlenker/E. Stephan/J. Wahl, Michelsberger Erdwerke im Raum Heilbronn. NeckarsulmObereisesheim 'Hetzenberg' und Ilsfeld 'Ebene', Lkr. Heilbronn, Heilbronn-Klingenberg 'Schlossberg', Stadkreis Heilbronn. Materialhefte zur Archäologie in Baden-Württemberg 81/3: Osteologische Beiträge (Stuttgart 2008) 166-248.

Steppan 2003 : K. Steppan, Taphonomie - Zoologie — Chronologie — Technologie — Ökonomie. Die Säugetierreste aus den jungsteinzeitlichen Grabenwerken in Bruchsal/Landkreis Karlsruhe. Materialhefte zur Archäologie in Baden-Württemberg 66 (Stuttgart 2003).

Stika 1996: H.-P. Stika, Vorgeschichtliche Pflanzenreste aus Heilbronn-Klingenberg: archäobotanische Untersuchungen zum Michelsberger Erdwerk auf dem Schlossberg (Bandkeramik, Michelsberger Kultur, Späthallstatt/Frühlatène). Materialhefte zur Archäologie in Baden-Württemberg 34 (Stuttgart 1996). 
Stuiver/Polach 1977: M. Stuiver /H. A. Polach, Reporting of ${ }^{14} \mathrm{C}$ data. Radiocarbon 19, 1977, 355-63.

Stuiver/Reimer 1986: M. Stuiver/P. J. Reimer, A computer program for radiocarbon age calculation. Radiocarbon 28, 1986, 1022-30.

Stuiver/Reimer 1993: M. Stuiver/P. J. Reimer, Extended ${ }^{14} \mathrm{C}$ data base and revised CALIB 3.0

${ }^{14} \mathrm{C}$ age calibration program. Radiocarbon 35 , 1993, 215-30.

Torke 2008: W. Torke, Fischreste aus dem Michelsberger Erdwerk Heilbronn-Klingenberg. In: U. Seidel, Michelsberger Erdwerke im Raum Heilbronn. Neckarsulm-Obereisesheim 'Hetzenberg' und Ilsfeld 'Ebene', Lkr. Heilbronn, Heilbronn-Klingenberg 'Schlossberg', Stadkreis Heilbronn. Band 1: Text, Literatur und Anhänge (Stuttgart 2008) 449-65.

Urton/Hobson 2005: E. J. Urton/K. A. Hobson, Intrapopulation variation in gray wolf isotope $\left(\delta^{15} \mathrm{~N}\right.$ and $\left.\delta^{13} \mathrm{C}\right)$ profiles: implications for the ecology of individuals. Oecologia 145, 2005, 31726.

Vandeputte et al. 1996: K. Vandeputte/L. Moens/R. Dams, Improved sealed-tube combustion of organic samples to $\mathrm{CO}_{2}$ for stable isotope analysis, radiocarbon dating and percent carbon determinations. Analytical Letters 29, 1996, 2761-73.

Wahl 2008: J. Wahl, Profan oder kultisch — bestattet oder entsorgt? Die menschlichen Skelettreste aus dem Michelsberger Erdwerken von Heilbronn-Klingenberg, NeckarsulmOberreisesheim und Ilsfeld. In: B. Schlenker/E. Stephan/J. Wahl, Michelsberger Erdwerke im Raum Heilbronn. Neckarsulm-Obereisesheim 'Hetzenberg' und Ilsfeld 'Ebene', Lkr. Heilbronn, Heilbronn-Klingenberg 'Schlossberg', Stadkreis Heilbronn. Band 3: Osteologische Beiträge (Stuttgart 2008) 703-848.

Ward/Wilson 1978: G. K. Ward /S. R. Wilson, Procedures for comparing and combining radiocarbon age determinations: a critique. Archaeometry 20, 1978, 19-31. 
Whittle et al. 2008: A. Whittle/A. Bayliss/F. Healy, The timing and tempo of change: examples from the fourth millennium cal BC in southern England. Cambridge Archaeological Journal 18, 2008, 65-70.

Whittle et al. 2011: A. Whittle/ F. Healy A. Bayliss, Gathering time: dating the Early Neolithic enclosures of southern Britain and Ireland (Oxford 2011). 